\title{
Spatial Structure of Stationary Nonequilibrium States in the Thermostatted Periodic Lorentz Gas.
}

\author{
F. Bonetto, \\ School of Mathematics, Georgia Institute of Technology, Atlanta GA 30332 \\ N. Chernov, A. Korepanov, \\ Department of Mathematics, University of Alabama at Birmingham, Birmingham AL 35294 \\ J.L. Lebowitz. \\ Departments of Mathematics and Physics, Rutgers University, Piscataway NJ 08854
}

February 22, 2018

\begin{abstract}
We investigate analytically and numerically the spatial structure of the non-equilibrium stationary states (NESS) of a point particle moving in a two dimensional periodic Lorentz gas (Sinai Billiard). The particle is subject to a constant external electric field $E$ as well as a Gaussian thermostat which keeps the speed $|\mathbf{v}|$ constant. We show that despite the singular nature of the SRB measure its projections on the space coordinates are absolutely continuous. We further show that these projections satisfy linear response laws for small $E$. Some of them are computed numerically. We compare these results with those obtained from simple models in which the collisions with the obstacles are replaced by random collisions. Similarities and differences are noted.
\end{abstract}

\section{Introduction}

In this paper we continue our study of nonequilibrium stationary states (NESS) maintained by a Gaussian thermostat [1, 4, 3, 9]. Theoretical analysis and computer simulations show that the NESS obtained from such artificial model dynamics can give useful information on real systems maintained in NESS by coupling with heat baths [8].

Here we focus on the Moran-Hoover $(\mathrm{MH})$ model of a single particle in a periodic billiard moving under the influence of an electric field $\mathbf{E}$ and a Gaussian thermostat that keeps the kinetic energy constant [12. The equations of motion are:

$$
\left\{\begin{array}{l}
\dot{\mathbf{x}}=\mathbf{p} \\
\dot{\mathbf{p}}=\mathbf{E}-\alpha(\mathbf{p}) \mathbf{p}+\mathbf{F}_{\text {obs }}(\mathbf{x}) \\
\alpha(\mathbf{p})=\frac{(\mathbf{p} \cdot \mathbf{E})}{(\mathbf{p} \cdot \mathbf{p})}
\end{array}\right.
$$

where $\mathbf{x}$ is the position, $\mathbf{p}$ the momentum of the particle with unit mass, and $\mathbf{F}_{\text {obs }}(\mathbf{x})$ represents elastic collision with the obstacles. It is clear from eq.(11) and the fact that collisions with the obstacles do not change $|\mathbf{p}|$ that $\frac{d}{d t}(\mathbf{p} \cdot \mathbf{p})=0$. We shall therefore set $|\mathbf{p}|=1$ from now on. 
The particle moves on a 2-dimensional torus whose side can be chosen to be 1 . An arrangement of the obstacles used for all the simulations presented in this paper is shown in Figure 1. The two obstacles have radii $r_{1}=0.2$ and $r_{2}=0.4$. This is also the arrangement used in our previous works [1, 2] and is chosen to have a finite horizon, i.e. there is an upper bound for the time between successive collisions of the particle with the obstacles. Moreover we take $\mathbf{E}$ to be along the horizontal $x$ axis, i.e. $\mathbf{E}=(E, 0)$. The analytical results apply to general geometries with finite horizons.

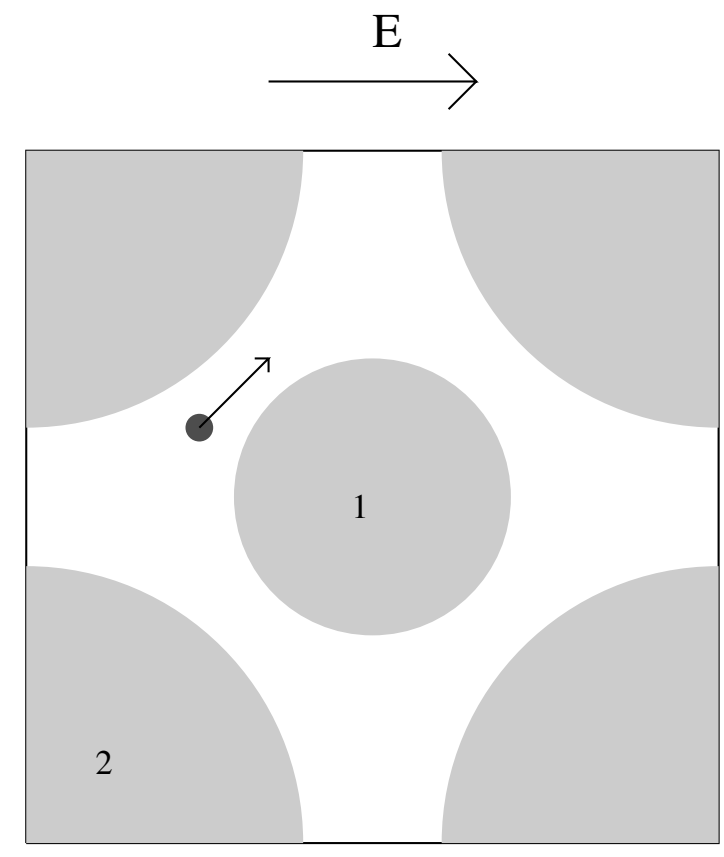

Figure 1: Typical obstacles placement.

The set of states where the particle collides with a given obstacle can be parametrized by two angles: $\vartheta \in[0,2 \pi]$ the angle on the obstacle between the collision point and the positive $x$ direction, and $\psi$ the angle between the particle velocity and the outgoing normal to the obstacle at the collision point. To obtain a complete coordinate system for the collision states we define the coordinate $\theta=\vartheta$ for obstacle 1 (see Figure 10 and $\theta=\vartheta+2 \pi$ for obstacle 2 so that $\theta \in[0,4 \pi]$. In these coordinates, the elastic collision is simply represented by the map $C(\theta, \psi)=(\theta, \pi-\psi)$, where $\psi \in[\pi / 2,3 \pi / 2]$ before collision and $\psi \in[-\pi / 2, \pi / 2]$ after collision. We will call $\mathcal{M}=[0,4 \pi] \times[-\pi / 2, \pi / 2]$ the set of possible pairs $(\theta, \psi)$ representing the position of the particle just after a collision. $\mathcal{M}$ corresponds to a Poincaré section of the flow. See Figure 2 for a depiction of $\theta$ and $\psi$.

Since $|\mathbf{p}|$ is constant, the trajectory of the particle can be represented by its position $\mathbf{x}(t)$ and the angle of its momentum with the horizontal axis $\phi(t)$. The motion of the particle between two collisions can be exactly integrated. Moreover one can construct the map $S_{E}(\theta, \psi)$ mapping the position and momentum of the particle just after a collision to its position and momentum just before the next collision, which may be with the same or a different obstacle.

In this way we can represent the dynamics in discrete time as the iteration of the map $T_{E}: \mathcal{M} \rightarrow \mathcal{M}$ between successive collisions given by $T_{E}=C \circ S_{E}$. Observe that this map is not continuous (grazing collisions) and that, for $E$ small, the dynamics is a perturbation of the free billiard dynamics 4 .

In our previous works we were primarily concerned with the SRB distribution associated 


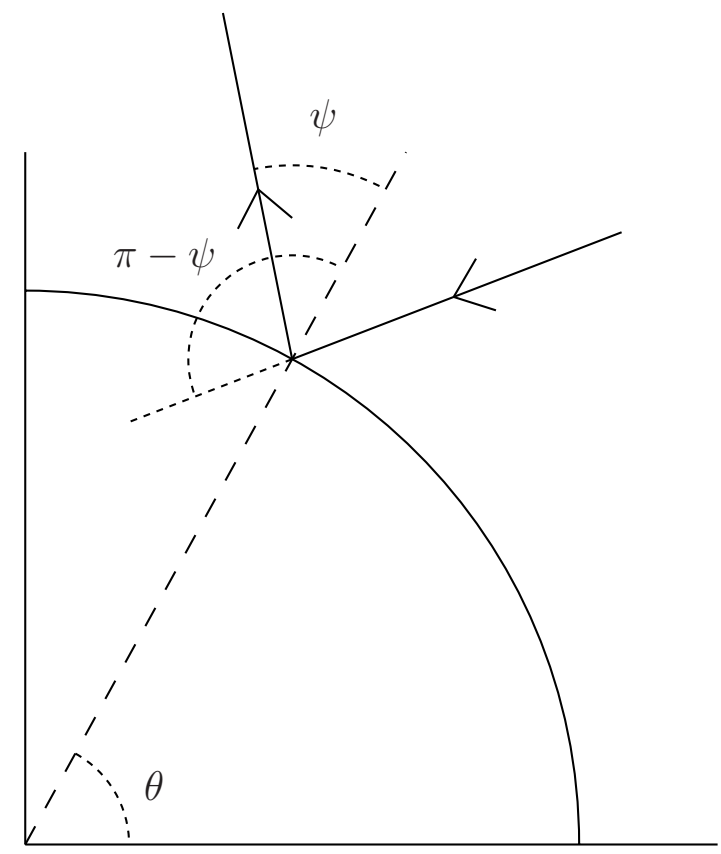

Figure 2: Elastic collision.

with $T_{E}$. Let $\mu_{0}$ be the measure on $\mathcal{M}$ given by

$$
\mu_{0}=\cos (\psi) \chi(\theta) d \theta d \psi / Z
$$

where $Z=4 \pi\left(r_{1}+r_{2}\right)$ is a normalization constant and $\chi(\theta)=r_{1}$ for $\theta \in[0,2 \pi]$ and $\chi(\theta)=r_{2}$ otherwise. Observe that $\mu_{0}$ is invariant under $T_{0}$. The SRB distribution $\mu_{E}(d \theta, d \psi)$ is defined as the weak limit of $\mu_{0}$ under the dynamics $T_{E}$, i.e.

$$
\mu_{E}=\mathrm{w}-\lim T_{E}^{n} \mu_{0} .
$$

The measure $\mu_{E}$, when it exists and is unique, represents the natural non equilibrium steady state (NESS) for the system [13. Clearly $\mu_{0}$ is the SRB measure of $T_{0}$.

From the SRB measure $\mu_{E}(d \theta, d \psi)$ on $\mathcal{M}$ for the collision map one can build the SRB measure $m_{E}(d \mathbf{x}, d \phi)$ on $M=Q \times[0,2 \pi]$ for the flow generated by eq.(1). Here $Q$ is $\mathbb{T} \backslash$ obstacles. This can be represented as:

$$
m_{E}(A)=\frac{1}{\bar{\tau}_{E}} \int_{\mathcal{M}} \int_{0}^{\tau_{E}(\theta, \psi)} I_{A}\left(\mathbf{X}_{t}^{E}(\theta, \psi), \Phi_{t}^{E}(\theta, \psi)\right) d t \mu_{E}(d \theta, d \psi)
$$

where $I_{A}$ is the indicator function of the set $A \subset M,\left(\mathbf{X}_{t}^{E}, \Phi_{t}^{E}\right)$ is the flow generated by eq.(1) and $\tau_{E}(\theta, \psi)$ is the time till the next collision when starting at $(\theta, \psi) \in \mathcal{M}$ with $\bar{\tau}_{E}=\int_{\mathcal{M}} \tau_{E}(\theta, \psi) \mu_{E}(d \theta, d \psi)$ denoting the mean free time.

In [4] it was proved that, for small fields $E,|E|<E_{0}$, the above model has a unique NESS described by an SRB measure $\mu_{E}$ which is singular with respect to the Liouville measure with Hausdorff dimension given by the standard Kaplan-Yorke formula [11.

The current $\mathbf{j}(E)$ in this NESS is given by

$$
\mathbf{j}(E)=m_{E}(\mathbf{v})
$$

where $\mathbf{v}=(\cos (\phi), \sin (\phi))$ is the velocity of the particle. This current was shown in [4] to be given by the Kawasaki formula cf. [6]. In the limit $E \rightarrow 0$ the Kawasaki formula reduces 
to the Green-Kubo formula for the conductivity $\kappa$ which satisfies the Einstein relation [4, 6]. An investigation of the current as a function of the field was carried out in [1]. It was argued there that the current is not a $C^{1}$ function of the field $E$ even close to $E=0$. The results of [4] were generalized in [9, 10, to systems where the collision rule or the free flow dynamics is perturbed.

In none of the above works was the spatial dependence of the singular (with respect to Lebesgue) measure $m_{E}(d \mathbf{x}, d \phi)$ studied. This is what we do in this note. We will describe analytical results and numerical studies of the spacial and angular dependence of the NESS $m_{E}(d \mathbf{x}, d \phi)$ when projected on $\phi \in[0,2 \pi]$ or on $\mathbf{x} \in Q$ and related quantities like the local average velocity.

The outline of the rest of the paper is as follows. In section 2 we introduce the local density, local average velocity and angular distribution derived from $m_{E}$. We find their dependence on position and field strength. We also show there computer generated pictures of the flow and compare them with the predictions of Green-Kubo formulas at small $E$. In section 3 we introduce and analyze two simple models in which the deterministic collisions with fixed obstacles are replaced by random collisions whose times form a Poisson process and compare their properties with those of the deterministic model. The appendixes are devoted to analytical justification of the claims in section 2. A paper describing results for the case where the system consists not just of one but of a large number of particles is in preparation.

\section{Local Structure of the SRB measure}

We define and study the several projections of the SRB measure $m_{E}$ introduced in the previous section. For clarity of exposition we delay derivations and justifications to the Appendices.

\subsection{Local Density and Average Velocity}

Two interesting quantities to study are the local density and local average velocity. More precisely, we define the projected measures on the position $\mathbf{x}$ as:

$$
\delta_{E}(A)=\int_{A \times[0,2 \pi)} m_{E}(d \mathbf{x}, d \phi)
$$

for any $A \subset Q$. This clearly defines a probability measure $\delta_{E}(d \mathbf{x})$ on $Q$. Using eq.(44), and defining

$$
J_{A}^{E}(\theta, \psi)=\frac{1}{\bar{\tau}_{E}} \int_{0}^{\tau(\theta, \psi)} I_{A}\left(\mathbf{X}_{t}^{E}(\theta, \psi)\right) d t
$$

where $I_{A}$ is the indicator function of the set $A$, we can represent $\delta_{E}(A)$ as the integral of a piecewise smooth function with respect to $\mu_{E}(d \theta, d \phi)$ :

$$
\delta_{E}(A)=\int_{\mathcal{M}} J_{A}^{E}(\theta, \psi) \mu_{E}(d \theta, d \psi)
$$

Observe that $J_{A}^{E}(\theta, \psi)$ is the relative amount of time the trajectory starting from $(\theta, \psi)$ spends in the set $A$, that is the amount of time divided by the mean free time $\bar{\tau}_{E}$.

We also define the vector measure for the local average velocity

$$
\nu_{E}(A)=\int_{A \times[0,2 \pi)}(\cos (\phi), \sin (\phi)) m_{E}(d \mathbf{x}, d \phi)
$$


Also this measure can be written as the integral of a piecewise smooth function with respect to $\mu_{E}(d \theta, d \phi)$, see eq.(52) below for details.

In Appendix $\mathrm{A}$ we show that, for $|E|<E_{0}$, the integrals in eq.(5) and eq.(8) define absolutely continuous measures. That is $\delta_{E}(d \mathbf{x})=n_{E}(\mathbf{x}) d \mathbf{x}$ and $\nu_{E}(d \mathbf{x})=n_{E}(\mathbf{x}) \mathbf{v}_{E}(\mathbf{x}) d \mathbf{x}$. We call $n_{E}(\mathbf{x})$ the local density and $\mathbf{v}_{E}(\mathbf{x})$ the local average velocity at $\mathbf{x}$. We show that both are continuous functions of both $\mathbf{x}$ and $E$ with $n_{0}(\mathbf{x})=$ const $=(\operatorname{Area}(Q))^{-1}$ and $\mathbf{v}_{0}(\mathbf{x})=0$.

To visualize the above numerically, we divided the torus of Figure 1 in a grid of $50 \times 50$ cells and computed the time average of the velocity of the particle when it crosses a cell. The results are shown in Figure 3. We also computed the local density on the same grid.

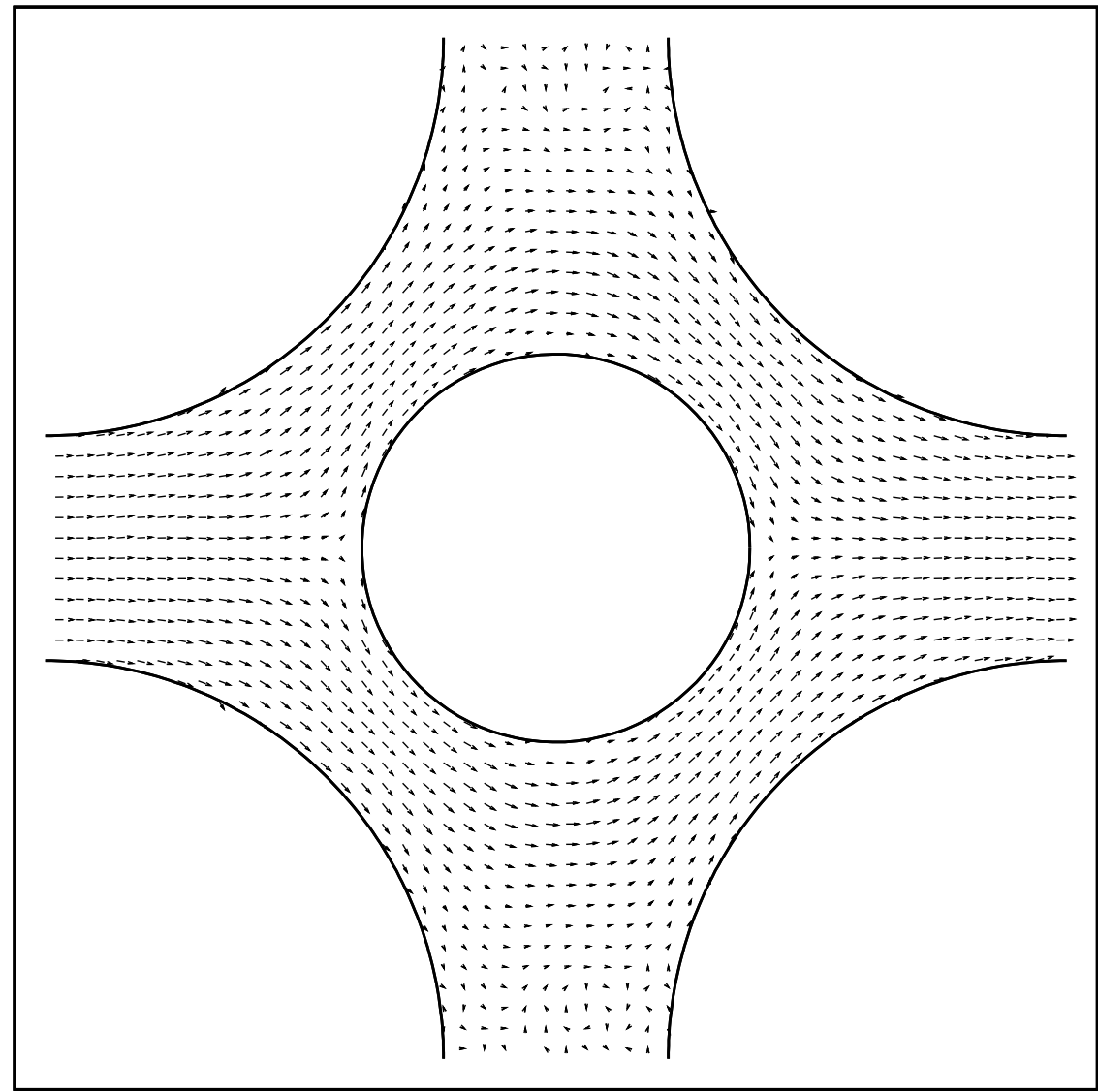

Figure 3: Average local velocity $\mathbf{v}_{E}(\mathbf{x})$ for $E=0.1$.

We now show that the local density $n_{E}(x)$ and the local average velocity $\mathbf{v}_{E}(\mathbf{x})$ are linear in the field $E$ when $E \rightarrow 0$, that is

$$
\begin{aligned}
& n_{E}(\mathbf{x})=n_{0}(\mathbf{x})+d(\mathbf{x}) E+o(E) \\
& \mathbf{v}_{E}(\mathbf{x})=\mathbf{k}(\mathbf{x}) E+o(E)
\end{aligned}
$$

where $d(\mathbf{x})$ and $\mathbf{k}(\mathbf{x})$ can be computed via Green-Kubo-type formulas as follows.

Consider the family of all velocity vectors originating at the point $\mathbf{x}$ (at which we are computing the density or average velocity); they make a one parameter family of phase states $W_{\mathbf{x}}=\{(\mathbf{x}, \phi) \mid 0<\phi<2 \pi\}$. Let $\rho^{\mathbf{x}}$ be the probability measure on $W_{\mathbf{x}}$ that has a uniform distribution over $\phi \in[0,2 \pi]$. We can map $W_{\mathbf{x}}$ to the collision space $\mathcal{M}$ by taking every point 
$(\mathbf{x}, \phi) \in W_{\mathbf{x}}$ to its first collision with $\partial Q$ in the past, under the field-free dynamics. The image of $W_{\mathbf{x}}$ will then be a collection $W_{0}$ of curves in $\mathcal{M}$ on which we get an induced probability measure $\rho_{0}$. Pulling this measure further back (into the past) we get a sequence of probability measures $\rho_{n}=T_{0}^{-n}\left(\rho_{0}\right)$, each sitting on a collection $W_{n}=T_{0}^{-n}\left(W_{0}\right)$ of curves in $\mathcal{M}$. With this definition we get that

$$
d(\mathbf{x})=c\left[\rho_{0}\left(\Delta_{0, \mathbf{x}}\right)+\sum_{n=1}^{\infty} \rho_{n}\left(\Delta_{0}\right)\right]
$$

where $c=\operatorname{Area}(Q)^{-1}=n_{0}(\mathbf{x}), \Delta_{0}=\tau_{0}(\theta, \psi) \cos (\theta+\psi)$ is the $x$-distance form the collision point $(\theta, \psi) \in \mathcal{M}$ to the next collision point and $\Delta_{0, \mathbf{x}}$ is the $x$-distance from the collision point $(\theta, \psi) \in \mathcal{M}$ to the point $\mathbf{x}$. Observe that $\rho_{0}$ is supported on points whose trajectory passes through $\mathbf{x}$ before colliding again. The above series converges exponentially, because the measures $\rho_{n}$ converge exponentially fast to the measure $\mu_{0}$ on $\mathcal{M}$ (see Theorem 7.31 in [5]) and $\mu_{0}\left(\Delta_{0}\right)=0$.

Consider now the two signed measures $\rho^{c, \mathbf{x}}$ and $\rho^{s, \mathbf{x}}$ on $W_{\mathbf{x}}$ that have densities $\cos \phi$ and $\sin \phi$, respectively, with respect to $\rho^{\mathbf{x}}$. As before, we can map $\rho^{c, \mathbf{x}}$ and $\rho^{s, \mathbf{x}}$ on the collision space $\mathcal{M}$ and obtain signed measures $\rho_{0}^{c}$ and $\rho_{0}^{s}$ on $W_{0}$, respectively. We also denote their images by $\rho_{n}^{c, s}=T_{0}^{-n}\left(\rho_{0}^{c, s}\right)$ on $W_{n}$ for $n \in \mathbb{Z}$.

$$
\mathbf{k}(\mathbf{x})=\frac{1}{2} \sum_{n=-\infty}^{\infty}\left(\rho_{n}^{c}\left(\Delta_{0}\right), \rho_{n}^{s}\left(\Delta_{0}\right)\right) .
$$

The terms in the series in eq.(12) converge to zero as $n \rightarrow \pm \infty$ exponentially fast, because the measures $\rho_{n}^{c, s}$ converge to the zero measure; this again follows from Theorem 7.31 in [5].

We note that the perturbation of the density $n_{E}(\mathbf{x})$ and of the local average velocity $\mathbf{v}_{E}(\mathbf{x})$ are linear in $E$, to the leading order, and the factor of $E$ is given, in both cases, by an infinite sum of correlations, i.e. the right hand sides of eq.(11) and eq.(12).

We computed numerically the coefficients $d$ and $\mathbf{k}$ in eq.(9) and eq.(10) to compare their predictions with the simulation results shown in Figure 3. We truncated the infinite sums in eqs.(1112) to $|n|<15$ since we saw no visible difference arise from taking more terms into consideration. Let $l_{x}^{+}=\{(x, y) \in Q\}$ be the vertical cross section placed at horizontal coordinate $x$ and $l_{y}^{-}=\{(x, y) \in Q\}$ be the horizontal cross section placed at vertical coordinate $y$. Finally let $\mathbf{e}_{x}=(1,0)$ and $\mathbf{e}_{y}=(0,1)$ be the unit vectors in the horizontal and vertical direction respectively. Figure 4 shows a comparison of the horizontal component $\left(\mathbf{v}_{E}(\mathbf{x}) \cdot \mathbf{e}_{x}\right)$ of $\mathbf{v}_{E}(\mathbf{x})$ along $l_{0.41}^{+}$with the prediction of eq.(10). In the same way, Figure 5 shows a comparison of the vertical component $\left(\mathbf{v}_{E}(\mathbf{x}) \cdot \mathbf{e}_{y}\right)$ of $\mathbf{v}_{E}(\mathbf{x})$ along $l_{0.41}^{-}$again with the prediction of eq.(10). In both figures the pluses represent the results of direct simulation while the crosses are obtained using the Green-Kubo formula eq.(12).

The comparison of $n_{E}(\mathbf{x})$ with eq.(9) is more difficult. Calling $n_{E}^{o}(\mathbf{x})=\left(n_{E}(\mathbf{x})-\right.$ $\left.n_{-E}(\mathbf{x})\right) / 2$ and $n_{E}^{e}(\mathbf{x})=\left(n_{E}(\mathbf{x})+n_{-E}(\mathbf{x})\right) / 2-n_{0}(\mathbf{x})$, we have that $n_{E}^{o}(\mathbf{x})$ satisfies the same linear response formula eq. (99) of $n_{E}(\mathbf{x})$ with the same coefficient $d(\mathbf{x})$ but we expect the remainder to be smaller. This is relevant in the present case since $n_{E}^{e}(\mathbf{x})$ and $n_{E}^{o}(\mathbf{x})$ appear to be of comparable magnitude. We observe that, due to the symmetry of the problem, $n_{E}(1-x, y)=n_{-E}(x, y)$ so that $n_{E}^{o}(x, y)=\left(n_{E}(x, y)-n_{E}(1-x, y)\right) / 2$. Figure 6 compares $n_{E}^{o}(\mathbf{x})$ along $l_{0.41}^{+}$with eq.(9). Again the pluses represents direct simulation while the crosses are obtained using the Green-Kubo formula eq.(11).

More generally, given a probability measure $\rho(d \mathbf{x}, d \phi)=l(\mathbf{x}, \phi) d \mathbf{x} d \phi$ absolutely continuous with respect to the Lebesgue measure on $Q$ let $\rho_{t}^{E}(d \mathbf{x}, d \phi)=l_{t}^{E}(\mathbf{x}, \phi) d \mathbf{x} d \phi$ be its time evolution with respect to the dynamics generated by eq.(11). In a similar way as above, we can then define: 


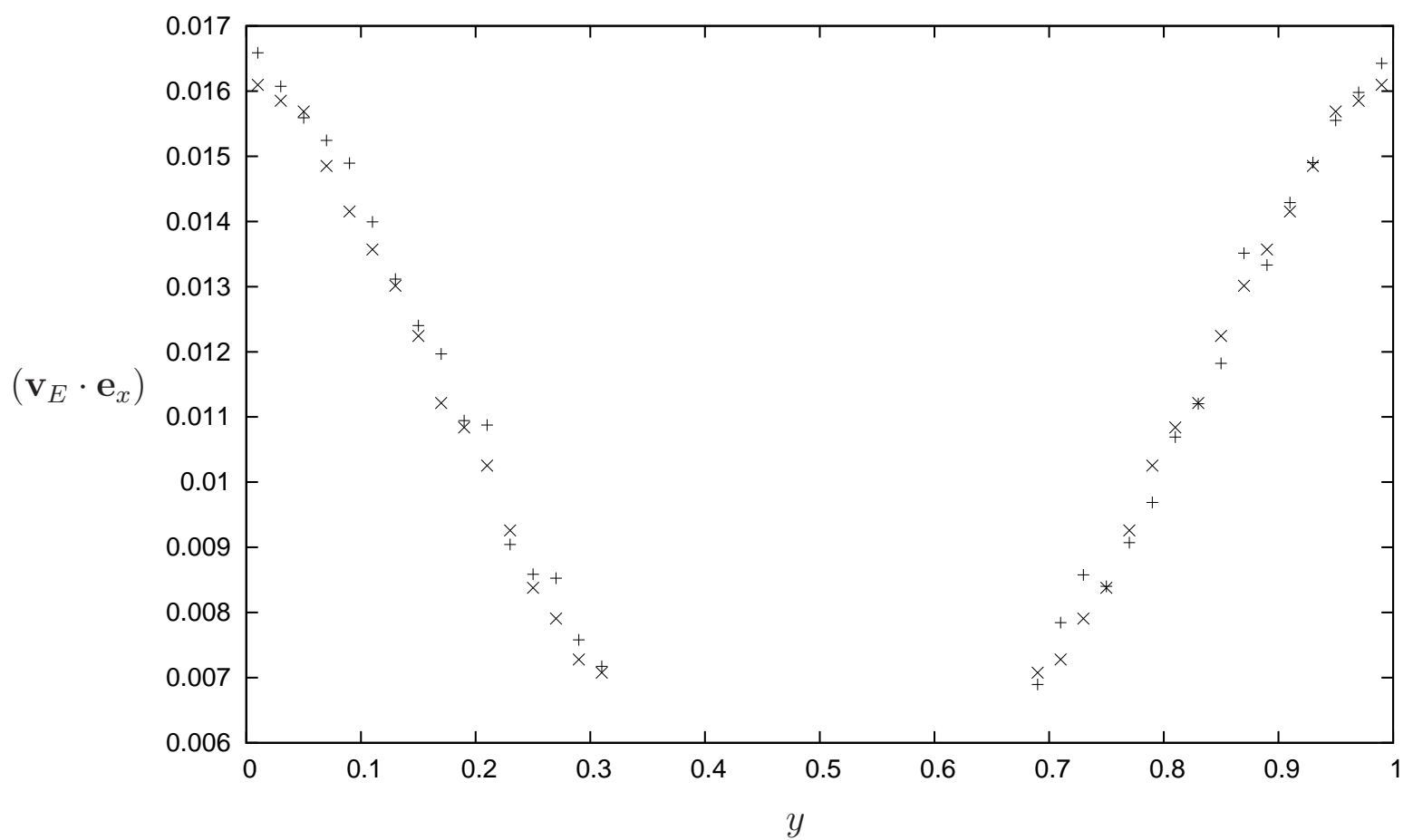

Figure 4: The $x$ component of the average local velocity $\mathbf{v}_{E}(\mathbf{x})$ for $E=0.1$ and $x=0.41$.

$$
\begin{aligned}
n_{t}^{E}(\mathbf{x}) & =\int l_{t}^{E}(\mathbf{x}, \phi) d \phi \\
n_{t}^{E}(\mathbf{x}) \mathbf{v}_{t}^{E}(\mathbf{x}) & =\int(\cos (\phi), \sin (\phi)) l_{t}^{E}(\mathbf{x}, \phi) d \phi
\end{aligned}
$$

The density $n_{t}^{E}(\mathbf{x})$ clearly satisfies a conservation law:

$$
\frac{d}{d t} \int_{A} n_{t}^{E}(x) d x=-\int_{\partial A} n_{t}^{E}(\mathbf{x})\left(\mathbf{v}_{t}^{E}(\mathbf{x}) \cdot \hat{\mathbf{n}}(\mathbf{x})\right) d \sigma(\mathbf{x})
$$

where $A$ is a subset of $Q$ with smooth enough boundary, $\hat{\mathbf{n}}(\mathbf{x})$ is the unit outward normal to $\partial A$ at $\mathbf{x}$ and $\sigma(\mathbf{x})$ is the length element on $\partial A$.

Taking the limit $t \rightarrow \infty$ and assuming that $\lim _{t \rightarrow \infty} n_{t}^{E}(\mathbf{x})=n_{E}(\mathbf{x})$ and $\lim _{t \rightarrow \infty} \mathbf{v}_{t}^{E}(x)=$ $\mathbf{v}_{E}(\mathbf{x})$ we obtain

$$
\int_{\partial A} n_{E}(\mathbf{x})\left(\mathbf{v}_{E}(\mathbf{x}) \cdot \hat{\mathbf{n}}(\mathbf{x})\right) d \sigma(\mathbf{x})=0
$$

The above assumption is not trivial. It is easy to show that, if $\lim _{t \rightarrow \infty} n_{t}^{E}(\mathbf{x})$ exists, it has to equal $n_{E}(\mathbf{x})$. On the other hand, we do not have a proof for the existence of such a limit. A similar argument holds for $\mathbf{v}_{t}^{E}(\mathbf{x})$. A complete justification of eq. (15) will thus require further work but we certainly expect it to be true.

Nonetheless we can test the validity of eq.15) numerically. Due to the symmetry of $Q$ we have that the average current $\mathbf{j}(E)=(j(E), 0)$. Moreover, since the collision are elastic, $\mathbf{v}_{E}(\mathbf{x})$ is tangent to $\partial Q$ for $\mathbf{x} \in \partial Q$. It follows from this that

$$
\int_{l_{x}^{+}} n_{E}(\mathbf{x})\left(\mathbf{v}_{E}(\mathbf{x}) \cdot \mathbf{e}_{x}\right) d y \equiv j(E)
$$




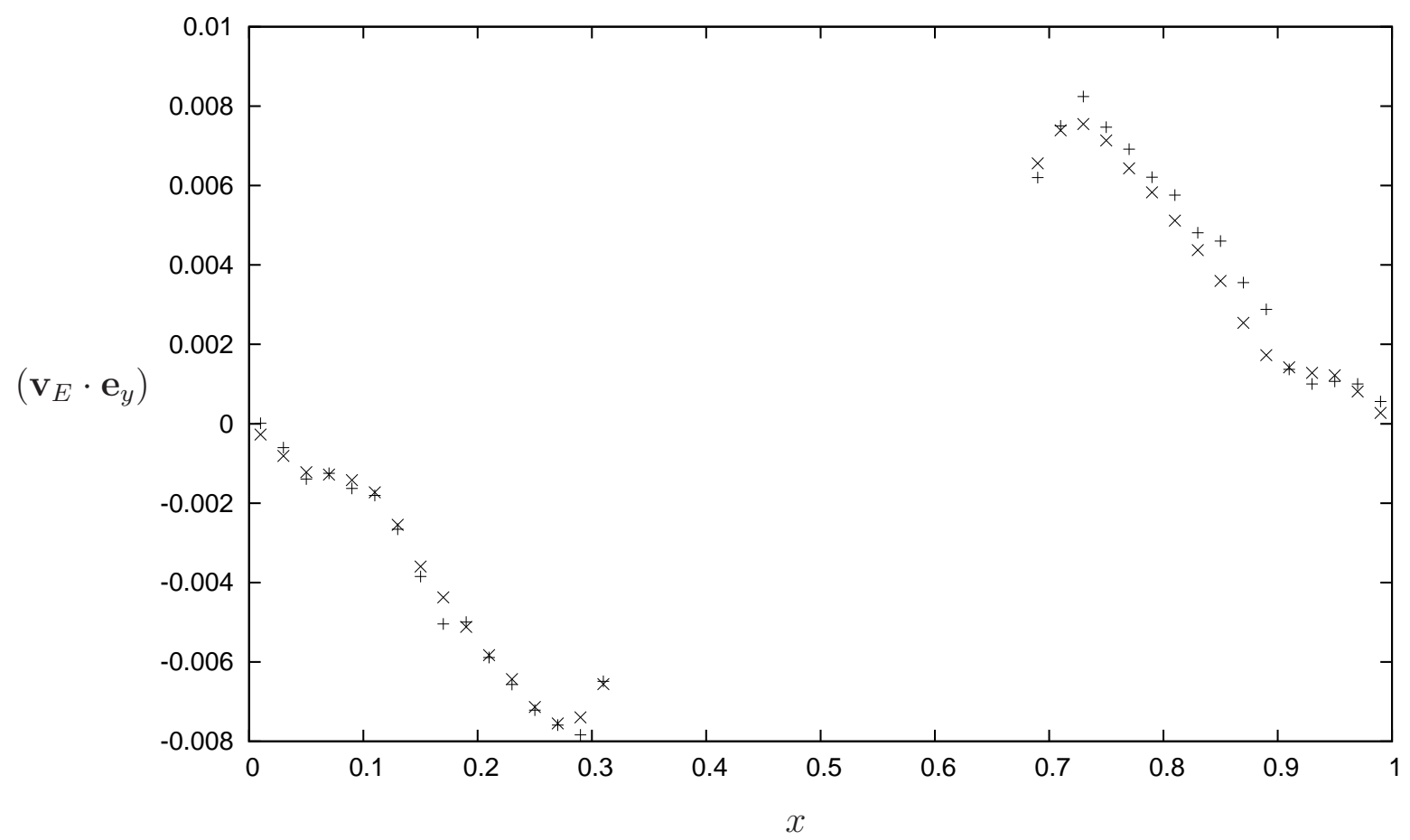

Figure 5: The $y$ component of the average local velocity $\mathbf{v}_{E}(\mathbf{x})$ for $E=0.1$ and $y=0.41$.

$$
\int_{l_{y}^{+}} n_{E}(\mathbf{x})\left(\mathbf{v}_{E}(\mathbf{x}) \cdot \mathbf{e}_{y}\right) d x \equiv 0
$$

independently on the value of $x$ or $y$. Both these equations are very well verified.

\subsection{Angular Distribution}

We now look at the projection of $m_{E}$ on the angle $\phi$. We can define the projected measure $\eta(d \phi)$ by setting, for a measurable set $A \subset[0,2 \pi]$,

$$
\eta_{E}(A)=\int_{M} I_{A}(\mathbf{x}, \phi) m_{E}(d \mathbf{x}, d \phi)
$$

where $I_{A}$ is the indicator function of the set $A$. Again we can write $\eta_{E}(A)$ as an integral on the SRB measure $\mu_{E}(d \theta, d \psi)$ as follows. Define the function:

$$
J_{A}^{E}(\theta, \psi)=\frac{1}{\bar{\tau}_{E}} \int_{0}^{\tau_{E}(\theta, \psi)} I_{A}\left(\Phi_{t}^{E}(\theta, \psi)\right) d t
$$

Then we have that

$$
\eta_{E}(A)=\int_{\mathcal{M}} J_{A}^{E}(\theta, \psi) \mu_{E}(d \theta, d \phi)=\mu_{E}\left(J_{A}^{E}\right)
$$

Using the argument in Appendix $\mathrm{A}$ we can show that, for $|E|<E_{0}, \eta_{E}$ is absolutely continuous with respect to $d \phi$, i.e. that $\eta_{E}(d \phi)=h_{E}(\phi) d \phi$ where $h_{E}(\phi)$ is a continuous function of both $\phi$ and $E$ with $h_{0}(\phi)=$ const $=1 / 2 \pi$, since the invariant measure $m_{0}$ is uniform on $Q \times[0,2 \pi]$.

We computed $h_{E}(\phi)$ numerically for $E=0.1$. The result is shown in Figure 7 , A striking characteristic of this distribution is the dip around $\phi=0$. This is somewhat unexpected since the effect of the field $E$ is to push the velocity of the particle to align with the positive $x$ 


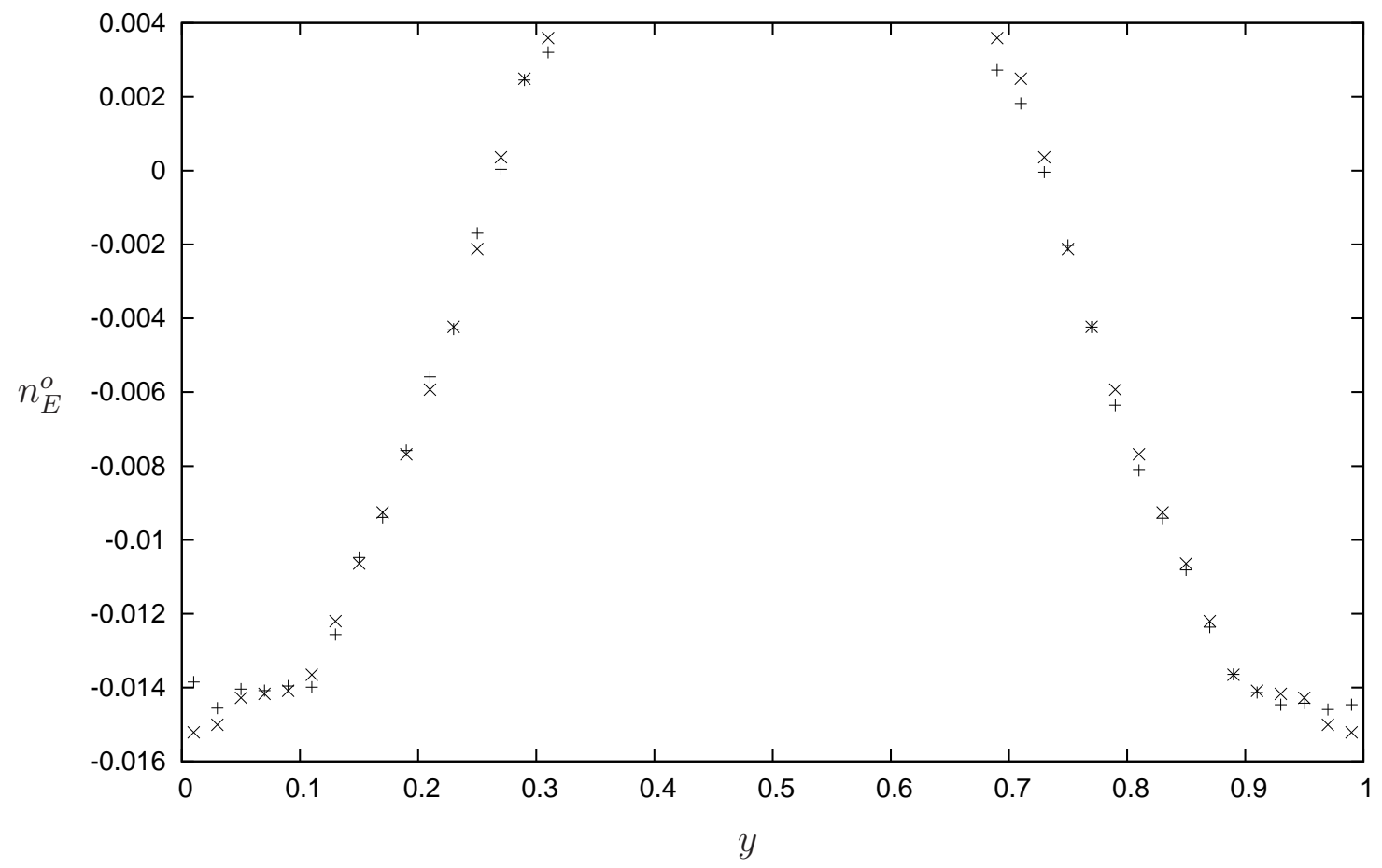

Figure 6: The symmetrized local density $n_{E}^{o}(\mathbf{x})$ for $E=0.1$ and $x=0.41$.

direction so that one would expect a maximum at $\phi=0$ rather than a local minimum (see also section 3 for a comparison with the stochastic models).

To understand this better we consider, for a given $\phi$, all points $(\theta, \psi) \in \mathcal{M}$ that produce the outgoing velocity vector $(\cos \phi, \sin \phi)$, i.e., we consider

$$
V_{\phi}=\{(\theta, \psi) \in \mathcal{M}: \psi+\theta=\phi(\bmod 2 \pi)\} .
$$

Now $\mathcal{M}$ is foliated by the lines $\left\{V_{\phi}\right\}, 0 \leq \phi<2 \pi$. Let $\mu_{0}^{\phi}$ denote the conditional measure induced by $\mu_{0}$ on the line $V_{\phi}$. If we use $\theta$ as the (only) coordinate on $V_{\phi}$, then

$$
d \mu_{0}^{\phi}=Z_{\phi}^{-1} \cos (\phi-\theta) \chi(\theta) d \theta
$$

where $Z_{\phi}$ is the normalizing factor

$$
Z_{\phi}=\int_{\cos (\phi-\theta)>0} \cos (\phi-\theta) \chi(\theta) d \theta=2\left(r_{1}+r_{2}\right) .
$$

We remind the reader that $\chi(\theta)=r_{1}$ and $0 \leq \theta<2 \pi$ on the first obstacle and $\chi(\theta)=r_{2}$ and $2 \pi \leq \theta<4 \pi$ on the second.

Now we consider the conditional distribution of the free flight time function $\tau_{0}$ on each line $V_{\phi}$. It turns out that its first moment is constant, i.e., $\mu_{0}^{\phi}\left(\tau_{0}\right)=\bar{\tau}_{0}$ for all $\phi$ 's, where $\bar{\tau}_{0}=\mu_{0}(\tau)$ is the total (unconditional) mean free time. In other words, the deterministic collision process is isotropic, on average. This seems to be a novel result in the studies of billiards and we prove it in Appendix C. We now argue that the observed dip near $\phi=0$, for small $E$ can be traced to the second moment, $\mu_{0}^{\phi}\left(\tau_{0}^{2}\right)$, which is not constant and which for our obstacles indeed has a local minimum at $\phi=0$.

We will show that the density $h_{E}(\phi)$ satisfies

$$
h_{E}(\phi)=\frac{1}{2 \pi}+a(\phi) E+o(E)
$$




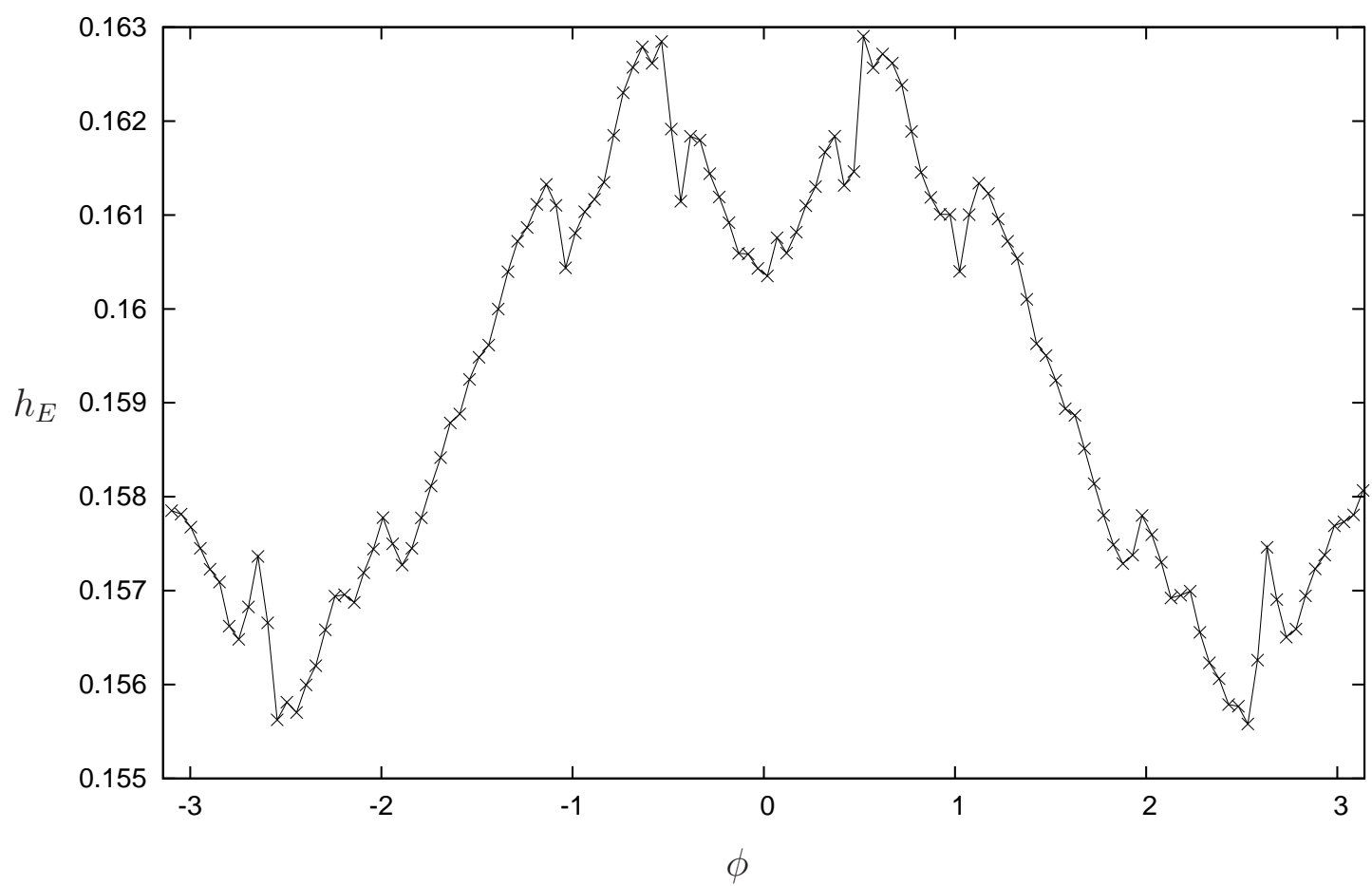

Figure 7: Angular distribution for $E=0.1$.

where $a(\phi)$ is given by a Green-Kubo formula

$$
a(\phi)=\frac{Z_{\phi}}{Z} \frac{1}{2 \bar{\tau}_{0}} \sum_{n=-\infty}^{\infty} \mu_{0}^{\phi}\left(\tau_{0} \cdot\left(\Delta_{0} \circ T_{0}^{n}\right)\right) .
$$

Recalling that $Z=4 \pi\left(r_{1}+r_{2}\right)$ is the normalization of $\mu_{0}$, see text after eq.(2), and $Z_{\phi}=$ $2\left(r_{1}+r_{2}\right)$ is independent of $\phi$, see eq. (17), we have that $Z_{\phi} / Z=1 / 2 \pi$. Again we see that the fluctuations of the density $h_{E}$ are linear in $E$, to the leading order, and the factor of $E$ is given by an infinite sum of correlations. The latter converges exponentially fast according to general results (Theorem 7.31 in [5]).

Usually its central term $(n=0)$ is the most significant, and it is given by

$$
\frac{Z_{\phi}}{Z} \frac{1}{2 \bar{\tau}_{0}} \mu_{0}^{\phi}\left(\tau_{0} \Delta_{0}\right)=\frac{\cos \phi}{4 \pi \bar{\tau}_{0}} \mu_{0}^{\phi}\left(\tau_{0}^{2}\right)
$$

The central term explicitly involves the second moment of $\tau_{0}$ restricted to $V_{\phi}$. Even though $\cos \phi$ has a maximum at $\phi=0$, it may be more than counterbalanced by a dip that the second moment $\mu_{0}^{\phi}\left(\tau_{0}^{2}\right)$ has near $\phi=0$. This is exactly what happens in our model shown in Figure 1 .

To check numerically the above results we proceed like in the case of $n_{E}(\mathbf{x})$ in Figure 7. We introduce the odd part of the angular distribution $h_{E}^{o}(\phi)=\left(h_{E}(\phi)-h_{-E}(\phi)\right) / 2$ and observe that it satisfies the linear response equation

$$
h_{E}^{o}(\phi)=a(\phi)+o(E)
$$

with $a(\phi)$ still given by eq. (19). Again we expect the reminder to be smaller. Finally, due to the symmetry of our system, we have that $h_{E}^{o}(\phi)=\left(h_{E}(\phi)-h_{E}(\phi+\pi)\right) / 2$.

Figure 8 presents the plot of eq.(21) and the numerically computed plot of $h_{E}^{o}(\phi)$ for $E=0.1$. The crosses represent the numerically computed value of $h_{E}^{0}(\phi)$. The pluses come 
from the central term of eq.(19). Already at this level the dip is clearly visible and the agreement is pretty good. Finally the boxes represent eq. (19) truncated at $|n|=20$. We have computed eq.(19) truncating the sum up to $|n|=100$ but no significant difference from $|n| \leq 20$ can be observed. This is clearly consistent with a fast convergence in the sum in eq.(19).

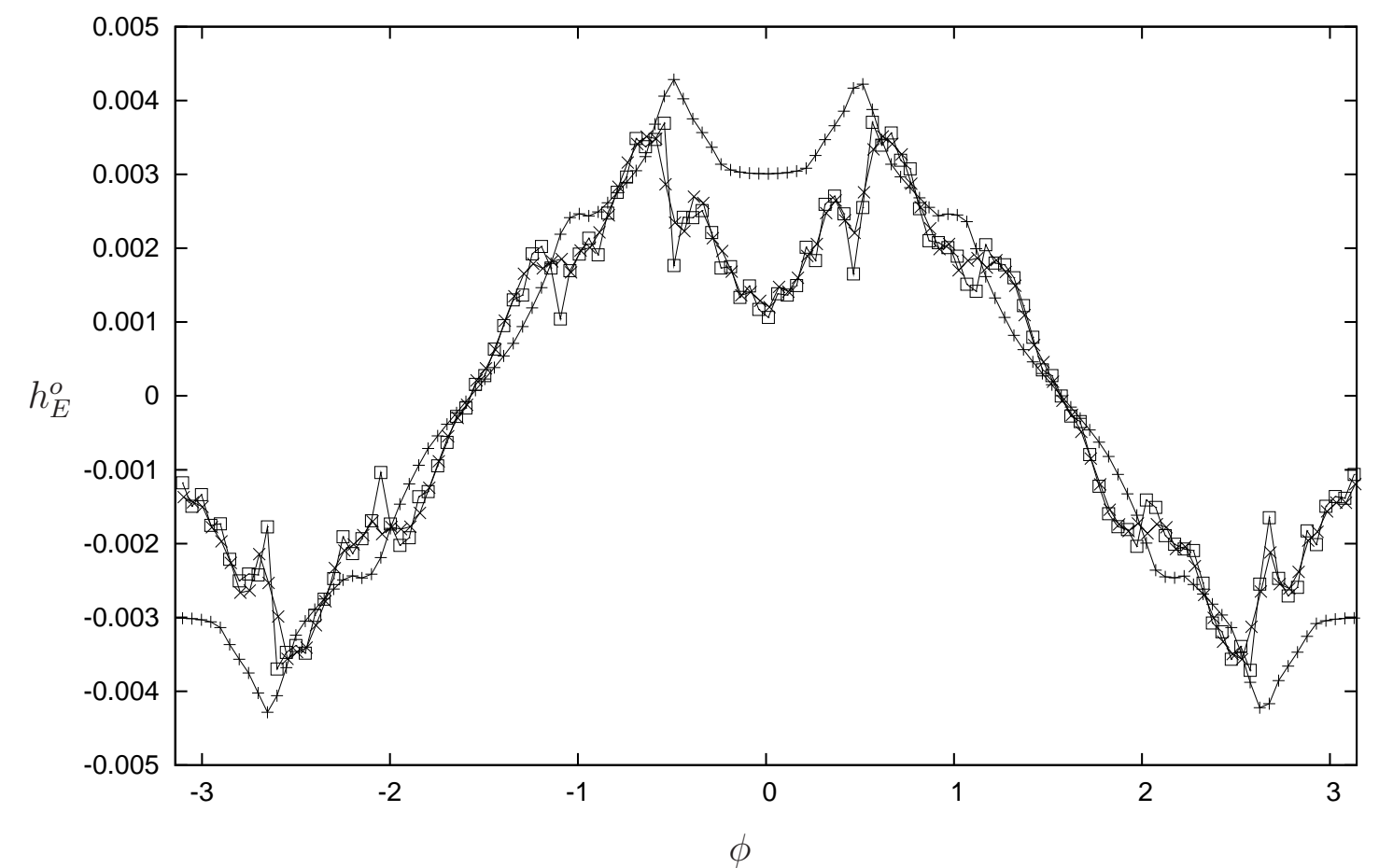

Figure 8: Comparison between the angular distribution for $E=0.1$ and eq.(59); see details after eq.(20).

Our analysis indicates that the dip at $\phi=0$ appears to be an artifact of the geometry of the scatterers chosen for our deterministic model.

\section{Random Collision Models}

In 2, we introduced a simplified version of the MH model by replacing the collisions with the fixed obstacles with a Poisson random collision process. More precisely we assume that, in every time interval $d t$ the particle has a probability equal to $\lambda|\mathbf{p}| d t$ (with $|\mathbf{p}|$ in this case fixed to be 1) to undergo a collision. Between collisions the particle moves according to eq.(11) without $F_{\text {obs }}$. When a collision happens we consider two collision rules:

I the velocity of the particle after the collision is in direction $\phi \in[0,2 \pi]$ with probability density $d \phi / 2 \pi$; or

II an angle $\eta \in[-\pi / 2, \pi / 2]$ is chosen at random with probability proportional to $\cos (\eta) d \eta$ and the direction of the velocity is changed according to an elastic collision rules for a particle colliding with an obstacle with outgoing velocity forming an angle $\eta$ with the normal to the obstacle.

We call the models with the above collision rules Model I and Model II.

We can think of Model II as representing a situation in which we have $N$ scatterers with diameter $\epsilon$ randomly placed in $\mathbb{T}$ and we consider the (Boltzmann-Grad) limit in which $N \rightarrow \infty, \epsilon \rightarrow 0$, such that $N \epsilon^{2} \rightarrow 0$ while $N \epsilon \rightarrow \lambda^{-1}$, the mean free path [7]. 
Let $f_{\alpha}(E, \mathbf{x}, \phi, t)$ be the probability density at time $t$ of finding the particle at $x$ with momentum $p=(\cos \phi, \sin \phi)$. Here $\alpha=\mathrm{I}, \mathrm{II}$ indicates Model I or Model II respectively. This density satisfies the equation:

$$
\begin{array}{r}
\partial_{t} f_{\alpha}(E, \mathbf{x}, \phi, t)-\mathbf{p} \partial_{\mathbf{x}} f_{\alpha}(E, \mathbf{x}, \phi, t)-E \partial_{\theta}\left(\sin \theta f_{\alpha}(E, \mathbf{x}, \phi, t)\right)= \\
\lambda\left(\int_{-\frac{\pi}{2}}^{\frac{\pi}{2}} p_{\alpha}(\eta) f_{\alpha}(E, \mathbf{x}, \phi+2 \eta+\pi, t) d \eta-f_{\alpha}(E, \mathbf{x}, \phi, t)\right)
\end{array}
$$

where, $t \in \mathbb{R}^{+}, \mathbf{x} \in \mathbb{T}$, the unit torus, $\mathbf{E}=(E, 0)$ is in the horizontal direction and $\lambda$ is the collision rate. Moreover we have $p_{\mathrm{I}}(\eta)=\pi^{-1}$ for Model I and $p_{\mathrm{II}}(\eta)=\cos \eta / 2$ for Model II.

It follows from eq.(22) that, when the distribution at time $0, f_{\alpha}(E, x, \phi, 0)$ does not depend on $\mathbf{x}$, the density $f_{\alpha}(E, \mathbf{x}, \phi, t)$ will also not depend on $\mathbf{x}$ for every $t>0$. Even if the initial state does depend on $x$, it is easy to show [3] that as $t \rightarrow \infty$ the system will approach a stationary density $f_{\alpha}(E, \phi)$ which will satisfy the equation:

$$
-\frac{E}{\lambda} \partial_{\phi}\left(\sin \phi f_{\alpha}(E, \phi)\right)=\int_{-\frac{\pi}{2}}^{\frac{\pi}{2}} p_{\alpha}(\eta) f_{\alpha}(E, \phi+2 \eta+\pi) d \eta-f_{\alpha}(E, \phi) .
$$

From now on we will set $\lambda=1$ since the stationary $f_{\alpha}$ depends only on $E / \lambda$.

We can try to solve this equation as a power series in $E$. Since $E$ is a singular perturbation the series will not be convergent for any non zero value of $E$. However, we expect that it will be an asymptotic series and accurate for small $|E|$. Writing

$$
f_{\alpha}(E, \phi)=\sum_{i=0}^{\infty} E^{i} f_{\alpha}^{(i)}(\phi)
$$

yields a hierarchy of equations for $i=0,1,2 \ldots$ :

$$
-\partial_{\phi}\left(\sin \phi f_{\alpha}^{(i-1)}(\phi)\right)=\int_{-\frac{\pi}{2}}^{\frac{\pi}{2}} p_{\alpha}(\eta) f_{\alpha}^{(i)}(\phi+2 \eta+\pi) d \eta-f_{\alpha}^{(i)}(\phi)
$$

with $f_{\alpha}^{(-1)} \equiv 0$. The equation for $i=0$ is easily solved and gives, as the unique solution, $f_{\alpha}^{(0)} \equiv 1$, since we require $\int f_{\alpha}(E, \phi) d \phi=2 \pi$. To solve the higher order equations we write

$$
f_{\alpha}^{(i)}(\phi)=\sum_{n=-\infty}^{\infty} \hat{f}_{\alpha}^{(i)}(n) \cos (n \phi)
$$

where we used the symmetry with respect to the direction orthogonal to the field to eliminate the terms in $\sin (n \theta)$ and clearly $f_{\alpha}^{(i)}(n)=f_{\alpha}^{(i)}(-n)$. In this way, for $n \neq 0$, eq. (25) becomes

$$
\hat{f}_{\alpha}^{(i)}(n)=\frac{n}{2}\left(1-\hat{p}_{\alpha}(n)\right)\left(\hat{f}_{\alpha}^{(i-1)}(n-1)-\hat{f}_{\alpha}^{(i-1)}(n+1)\right)
$$

with $\hat{p}_{\mathrm{I}}(n)=0$ for Model I and $\hat{p}_{\mathrm{II}}(n)=1 / 4 n^{2}$ for Model II. Finally $f_{\alpha}^{(0)}(n)=\delta_{n, 0}$, again due to the normalization condition. This yields

$$
\begin{aligned}
f_{\mathrm{I}}(E, \phi) & =1+E \cos (\phi)+E^{2} \cos (2 \phi)+\ldots \\
f_{\mathrm{II}}(E, \phi) & =1+\frac{3}{4} E \cos (\phi)+\frac{45}{64} E^{2} \cos (2 \phi)+\ldots
\end{aligned}
$$

We can compare the above results with numerical simulation of the stochastic processes generating eq.(23). We set $E=0.2$ and run both processes for $10^{8}$ collisions. The results 


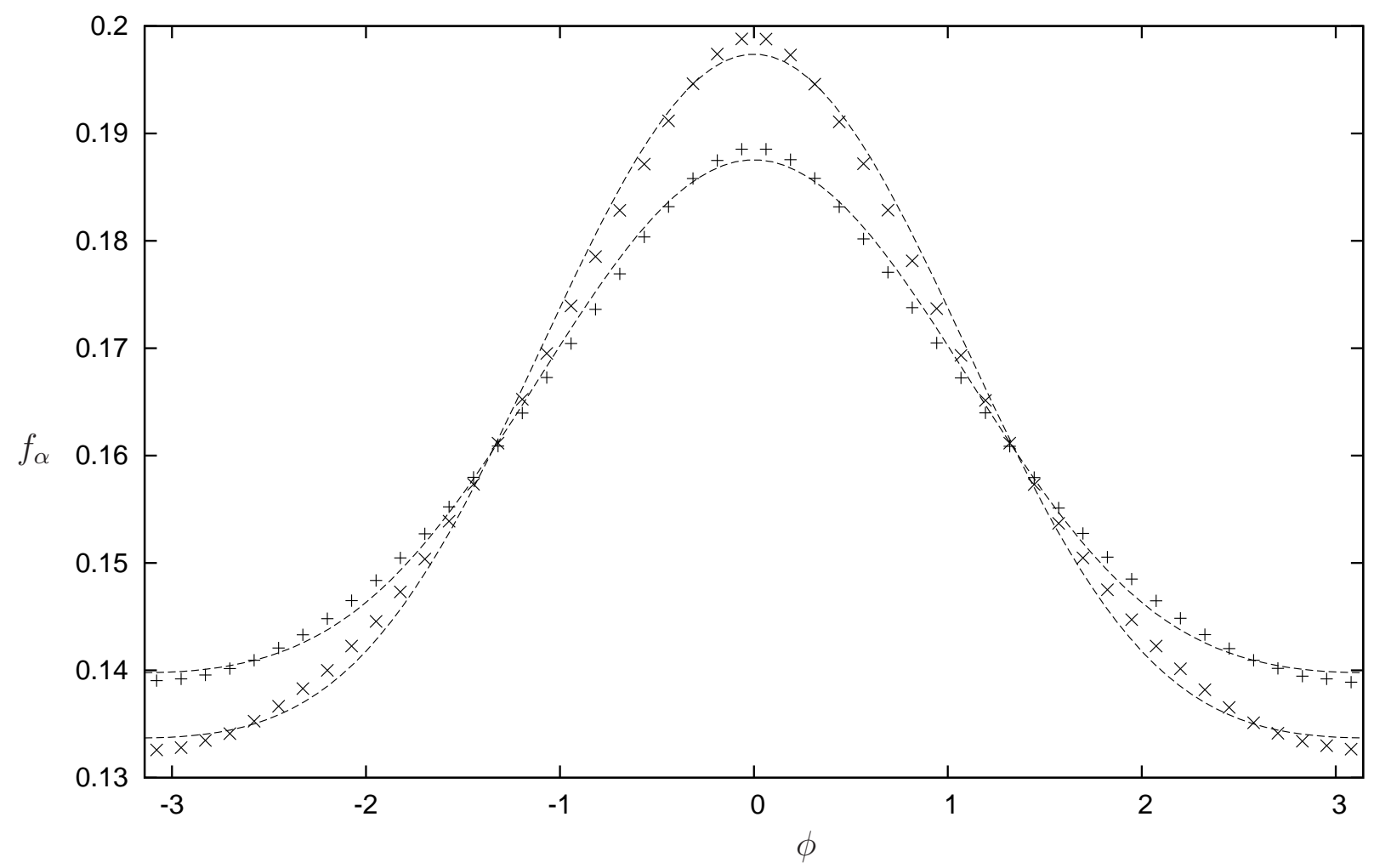

Figure 9: Comparison between numerical simulations of the stochastic process and the power series expansion eq.(27) for $E=0.2$. See explanation after eq.(27).

are plotted in Figure 9. The crosses refer to Model I while the pluses refer to Model II. Superimposed are the graph obtained from eq.(27). As one can see, the fit is very good. This is in agreement with our expectation that the series in $E$ is an asymptotic one. In the case of model I this can be rigorously justified, see eq.(30) below.

We note that, for both Models, the power series for $f_{\alpha}(0.2, \phi)$ has a global maximum for $\phi=0$. Since this is not true for the angular distribution of the deterministic MH model, see section 2.2, we will investigate the behavior of $f(E, \psi)$ near $\phi=0$ more closely.

\subsection{Model I}

Eq.(23) can be written as:

$$
-E \partial_{\phi}\left(\sin \phi f_{\mathrm{I}}(E, \phi)\right)=1-f_{\mathrm{I}}(E, \phi)
$$

where we normalize $f_{\mathrm{I}}$ as $\int f_{\mathrm{I}}(E, \phi) d \phi=2 \pi$.

Eq.(28) can be solved by introducing the function

$$
h(E, \phi)=\left(\frac{1-\cos \phi}{1+\cos \phi}\right)^{\frac{1}{2 E}}
$$

which is a solution of the differential equation $\partial_{\phi} h(E, \phi)=\frac{h(E, \phi)}{E \sin \phi}$, and defining

$$
f_{\mathrm{I}}(E, \phi)=\frac{h(E, \phi)}{\sin \phi} g(E, \phi)
$$

Substituting in eq.(28) we obtain 


$$
\partial_{\phi} g(E, \phi)=-h(E, \phi+\pi)
$$

Observe that $h(E, \phi)$ has a non integrable singularity at $\phi=\pi$ so that, for $f_{\mathrm{I}}(E, \phi)$ to be integrable we need $g(E, \pi)=0$. We can thus represent the solution as:

$$
f_{\mathrm{I}}(E, \phi)=\frac{h(E, \phi)}{E \sin (\phi)} \int_{\phi}^{\pi} h(E, \eta+\pi) d \eta
$$

We list below some properties of $f_{\mathrm{I}}(E, \phi)$ that will be useful in the following. We have two possible situations:

$E<1$ : In this case $f_{\mathrm{I}}(E, \phi)$ is continuous in $\phi$ for every $\phi$. Moreover it is easy to see that $f_{\mathrm{I}}(E, \phi)$ is $C^{\infty}$ for $\phi \neq 0, \pi$. For $\phi=0, \pi$, if $E<1 / n, f_{\mathrm{I}}(E, \phi)$ is $C^{n-1}$ and $\partial_{\phi}^{n} f_{\mathrm{I}}(E, \phi)$ is Hölder continuous of exponent $\alpha$ for $0<\alpha<1 / E-n$.

$E>1 \mathrm{In}$ this case $f_{\mathrm{I}}(E, \phi)$ is still $C^{\infty}$ everywhere but for $\phi=0, \pi$. At $\phi=0$ we have a singularity and $f_{\mathrm{I}}(E, \phi) \simeq \phi^{1 / E-1}$. More precisely the function $\phi^{1-1 / E^{\prime}} f_{\mathrm{I}}(E, \phi)$ is Hölder continuous of exponent $0<\alpha<1 / E-1 / E^{\prime}$, for every $E^{\prime}>E$.

Starting form eq.(29) and integrating by part we obtain:

$$
\begin{aligned}
f_{\mathrm{I}}(E, \phi) & =1-\frac{h(E, \phi)}{\sin (\phi)} \int_{\phi}^{\pi} \cos \eta h(E, \eta+\pi) d \eta= \\
& =1+E \cos \phi+E \frac{h(E, \phi)}{\sin (\phi)} \int_{\phi}^{\pi} \cos 2 \eta h(E, \eta+\pi) d \eta= \\
& =1+E \cos \phi+E^{2} \cos (2 \phi)+E^{2} \frac{h(E, \phi)}{\sin (\phi)} \int_{\phi}^{\pi} \partial_{\phi}[\cos 2 \eta \sin \eta] h(E, \eta+\pi) d \eta= \\
& =\sum_{i=0}^{N} E^{n} f_{\mathrm{I}}^{(i)}(\phi)+E^{N} R_{N}(E, \phi)
\end{aligned}
$$

The above expansion coincides with the one obtained in eqs.(24) 26). It is not difficult to see that $\left|R_{N}(E, \phi)\right| \leq K C^{N} N$ !. Since it is clear from eq.(29) that $f_{I}(E, \phi)$ is not analytic in $E$ for small $E$, this inequality means that, as we discussed previously, the perturbative series for $f_{\mathrm{I}}(E, \phi)$ is at least asymptotic. Notwithstanding this, eq(30) and the regularity properties of $f_{\mathrm{I}}(E, \phi)$ tell us that, for $E$ small, $f_{\mathrm{I}}(E, \phi)$ has a unique maximum at $\phi=0$ and a unique minimum at $\phi=\pi$.

\subsection{Model II}

We can use the solution of Model I to get more analytical information on Model II. Proceeding as in eq.(28) we write the solution of eq.(23) as

$$
f_{\mathrm{II}}(E, \phi)=\frac{h(E, \phi)}{E \sin \phi} g(E, \phi)
$$

and obtain the representation for $g(E, \phi)$ :

$$
\partial_{\phi} g(E, \phi)=-\frac{1}{4 E} h(E, \phi+\pi) \int_{-\pi}^{\pi}\left|\cos \left(\frac{\omega-\phi}{2}\right)\right| \frac{h(E, \omega+\pi)}{\sin (\omega+\pi)} g(E, \omega+\pi) d \omega
$$

from which, reasoning as in Model I, we get 


$$
f_{\mathrm{II}}(E, \phi)=\frac{1}{4 E \sin (\phi)} h(E, \phi) \int_{\phi}^{\pi} h(E, \eta+\pi) \int_{-\pi}^{\pi}\left|\cos \left(\frac{\omega-\eta}{2}\right)\right| f(E, \omega+\pi) d \omega d \eta
$$

for $0<\phi<\pi$. We can then set $f_{\mathrm{II}}(E,-\phi)=f_{\mathrm{II}}(E, \phi)$. Observe that the above equation can be written has as

$$
f_{\mathrm{II}}(E, \phi)=\int_{-\pi}^{\pi} Q(\phi, \omega) f_{\mathrm{II}}(E, \omega) d \omega
$$

where

$$
Q(\phi, \omega)=\frac{1}{4 E \sin (\phi)} h(E, \phi) \int_{\phi}^{\pi} h(E, \eta+\pi)\left|\sin \left(\frac{\omega-\eta}{2}\right)\right| d \eta
$$

for $0<\phi<\pi$ and $Q(-\phi, \omega)=Q(\phi, \omega)$. It is easy to see that $Q(\phi, \omega)>0$ for every $\phi, \omega$. Moreover we have

$$
\begin{aligned}
\int_{0}^{\pi} Q(\phi, \omega) d \phi & =\lim _{\epsilon \rightarrow 0} \int_{\epsilon}^{\pi-\epsilon} Q(\phi, \omega) d \phi= \\
& =\frac{1}{4} \int_{0}^{\pi} h(E, \phi) h(E, \phi+\pi)\left|\sin \left(\frac{\omega-\phi}{2}\right)\right| d \phi+ \\
& -\frac{1}{4} \lim _{\epsilon \rightarrow 0} h(E, \epsilon) \int_{\epsilon}^{\pi} h(E, \eta+\pi)\left|\sin \left(\frac{\omega-\eta}{2}\right)\right| d \eta \\
& +\frac{1}{4} \lim _{\epsilon \rightarrow 0} h(E, \pi-\epsilon) \int_{\pi-\epsilon}^{\pi} h(E, \eta+\pi)\left|\sin \left(\frac{\omega-\eta}{2}\right)\right| d \eta= \\
& =\frac{1}{4} \int_{0}^{\pi}\left|\sin \left(\frac{\omega-\phi}{2}\right)\right| d \phi
\end{aligned}
$$

where we have used that $h(E, \phi)=h(E, \phi+\pi)^{-1}$ and that, for $\epsilon$ small, $h(E, \epsilon) \simeq \epsilon^{1 / E}$ while $h(E, \epsilon+\pi) \simeq \epsilon^{-1 / E}$. Proceeding in the same way for $-\pi<\phi<0$, we get

$$
\int_{-\pi}^{\pi} Q(\phi, \omega) d \phi=\frac{1}{4} \int_{-\pi}^{\pi}\left|\sin \left(\frac{\omega-\phi}{2}\right)\right| d \phi=1
$$

for every $\omega$. Finally, in the same way we got the regularity properties of $f_{\mathrm{I}}(E, \phi)$, we can see that, if $E<1$ then $Q f_{\mathrm{II}}$ is a Hölder continuous function with Hölder norm bounded by the $L^{\infty}$ norm of $f_{\mathrm{II}}$. This immediately implies, by the Ascoli-Arzelá theorem, that $Q$ is a compact linear operator on $C^{0}$. In this situation we can apply the Krein-Rutman theorem, see [15, 16], and obtain that there is a unique function $f_{\mathrm{II}}(E, \phi)$ that satisfies eq. (33). Moreover $f_{\mathrm{II}}(E, \phi)>0$ for every $\phi$. A similar argument tells us that, for $E>1$, there is a unique solution of eq. (33) and it can be written as

$$
f_{\mathrm{II}}(E, \phi)=|\sin (\phi)|^{1-\frac{1}{E}} l(E, \phi)
$$

with $l(E, \phi)$ continuous in $\phi$ and strictly positive.

Observe that, for any integrable function $f(\phi)$, we have

$$
\begin{aligned}
\partial_{\phi} \int_{-\pi}^{\pi}\left|\cos \left(\frac{\omega-\phi}{2}\right)\right| f(\omega+\pi) d \omega & =\frac{1}{2} \int_{-\pi}^{\pi} \operatorname{sgn}(\omega-\phi)\left|\sin \left(\frac{\omega-\phi}{2}\right)\right| f(E, \omega+\pi) d \omega \\
\partial_{\phi}^{2} \int_{-\pi}^{\pi}\left|\cos \left(\frac{\omega-\phi}{2}\right)\right| f(E, \omega+\pi) d \omega & =-\frac{1}{4} \int_{-\pi}^{\pi}\left|\cos \left(\frac{\omega-\phi}{2}\right)\right| f(E, \omega+\pi) d \omega+f(\phi)
\end{aligned}
$$

Thus the above integral is always at least $C^{1}$, while if $f(\phi)$ is $C^{n}$ it is $C^{n+2}$. This implies that $f_{\mathrm{II}}(E, \phi)$ has the same regularity properties as a function of $\phi$ as $f_{\mathrm{I}}(E, \phi)$. In particular if 
$E \leq 1 / 3, f_{\mathrm{II}}(E, \phi)$ is $C^{2}$ and we can try to compute $f_{\mathrm{II}}^{\prime \prime}(E, 0)$ explicitly. Observe that eq.(23) tells us that

$$
E \sin \phi f_{\mathrm{II}}^{\prime}(E, \phi)+E \cos \phi f_{\mathrm{II}}(E, \phi)=-\frac{1}{4} \int_{-\pi}^{\pi}\left|\cos \left(\frac{\omega-\phi}{2}\right)\right| f_{\mathrm{II}}(E, \omega+\pi) d \omega+f_{\mathrm{II}}(E, \phi)
$$

Evaluating at $\phi=0$ we get

$$
f_{\mathrm{II}}(E, 0)=\frac{1}{4(1-E)} \int_{-\pi}^{\pi} \cos \left(\frac{\omega}{2}\right) f_{\mathrm{II}}(E, \omega+\pi) d \omega
$$

As expected, this equation loose meaning when $E \geq 1$ since $f_{\mathrm{II}}(E, \phi)$ is no more continuous at $\phi=0$. We can now differentiate both side of eq.(23) and obtain, after evaluating in $\phi=0$,

$$
(1-2 E) f_{\mathrm{II}}^{\prime}(E, \phi)=\frac{1}{8} \int_{-\pi}^{\pi} \sin \left(\frac{\omega}{2}\right) f_{\mathrm{II}}(E, \omega+\pi) d \omega=0
$$

for symmetry reasons. Again this equation make sense only if $E<1 / 2$. Finally, differentiating once more, we get

$$
3 E f_{\mathrm{II}}^{\prime \prime}(E, 0)-E f_{\mathrm{II}}(E, 0)=\frac{1}{16} \int_{-\pi}^{\pi} \cos \left(\frac{\omega}{2}\right) f_{\mathrm{II}}(E, \omega) d \omega-\frac{1}{4} f_{\mathrm{II}}(E, 0)+f_{\mathrm{II}}^{\prime \prime}(E, 0)
$$

Using eq.(36) we get

$$
f_{\mathrm{II}}^{\prime \prime}(E, 0)=-\frac{3}{4} \frac{E}{1-3 E} f_{\mathrm{II}}(E, 0)
$$

that is clearly negative for $E<1 / 3$ so that we have that $f(E, \phi)$ has a local maximum at $\phi=0$. Observe that expanding this formula to third order in $E$ we get a result in agreement with the expansion in eq.(27)

\section{Acknowledgment}

The authors thank Eric Carlen for many insightful comments and discussions. The work of FB was supported in part by NSF grant 0604518. The work of NC was supported in part by NSF grant DMS-0969187. The work of JLL was supported in part by NSF grant DMR08021220 and by AFOSR grant AF-FA9550-07. The authors are also grateful to the Alabama supercomputer administration for computational resources.

\section{Appendices}

\section{A Regularity of Projections of SRB measures}

SRB measures are characterized by absolutely continuous conditional distributions on unstable manifolds, but generally they are singular. Singularity of a measure $\mu$ means that there is a subset $\mathcal{M}^{\prime} \subset \mathcal{M}$ in the phase space $\mathcal{M}$ such that $\mu\left(\mathcal{M}^{\prime}\right)=1$ but the Lebesgue measure of $\mathcal{M}^{\prime}$ is zero. In that case $\mu$ does not have a density on $\mathcal{M}$.

However in physics one rarely observes measures on the entire phase space; it is more common to observe distributions of some selected variables (e.g., positions or velocities of 
selected particles). The distributions of those variables are obtained by projection of the relevant measure onto the corresponding variables. And the resulting distribution is often absolutely continuous, with a continuous density, despite the singularity of the measure in the whole of phase space.

Similar smoothness results hold if, instead of projecting the measure onto certain variable(s), we integrate some smooth functions with respect to all the other variables. For example, in eq.(8) we integrate $\cos \phi$ and $\sin \phi$ with respect to $\phi$ and get an absolutely continuous distribution with respect to $\mathbf{x}$ with a continuous density.

We sketch a proof here that in our Moran-Hoover model the corresponding projections have continuous bounded densities. Let $\mu_{E}$ denote the SRB measure on the collision space with coordinates $(\theta, \psi)$ for a given value of $E$. Since we will consider only a given value of $E$, we will suppress the dependence on $E$ in what follows. All estimates are uniform in $E$. Consider a projection of $\mu$ onto a line transversal (not parallel or perpendicular) to stable and unstable manifolds and singularity manifolds. For simplicity, let $\mu$ be projected onto the $\theta$ axis.

The density of the projection can be computed as

$$
\rho(\theta)=\lim _{\delta \rightarrow 0} \delta^{-1} \mu\left(R_{\theta, \delta}\right)
$$

where $R_{\theta, \delta}=\left\{\left(\theta^{\prime}, \psi\right): \theta<\theta^{\prime}<\theta+\delta\right\}$ is a rectangle in the collision space of size $\delta$ in the $\theta$ direction. It is known that the SRB measure $\mu$ satisfies

$$
\mu(r(\theta, \psi)<\delta)<C \delta \quad \forall \delta>0
$$

for some constant $C>0$ which is uniform for all small fields $E$, see [9]. Here $r(\theta, \psi)$ denotes the distance from $(\theta, \psi) \in \mathcal{M}$ to the nearer endpoint of the unstable manifold passing through $(\theta, \psi)$ (that manifold is a smooth curve which is divided by $(\theta, \psi)$ into two segments; so $r(\theta, \psi)$ denotes the length of the shorter one).

Now for each unstable manifold $W$, the intersection $W \cap R_{\theta, \delta}$ is a segment of $W$ that has length bounded above by $C^{\prime} \delta$ for some constant $C^{\prime}>0$. Hence its relative measure (within $W$ ) is of the same order as the measure of the segment of length $\delta$ at an endpoint of $W$. We recall that the conditional densities of SRB measures are Hölder continuous, and their fluctuations are uniformly bounded [5, 9, 10. Now eq. (38) implies

$$
m\left(R_{\theta, \delta}\right)<C^{\prime \prime} \delta
$$

for some constant $C^{\prime \prime}>0$, hence $\rho(\theta)$ is uniformly bounded.

Next we prove that $\rho(\theta)$ is continuous. For $\theta_{1} \approx \theta$ we have

$$
\rho\left(\theta_{1}\right)-\rho(\theta)=\lim _{\delta \rightarrow 0} \frac{\mu\left(R_{\theta_{1}, \delta}\right)-\mu\left(R_{\theta, \delta}\right)}{\delta}
$$

Now there are unstable manifolds that cross both rectangles $R_{\theta_{1}, \delta}$ and $R_{\theta, \delta}$ and those which cross only one of them; accordingly we have

$$
\mu\left(R_{\theta_{1}, \delta}\right)-\mu\left(R_{\theta, \delta}\right)=\Delta_{1}+\Delta_{2}
$$

where $\Delta_{1}$ accounts for the former, and $\Delta_{2}$ for the latter. The conditional density of $\mu$ on each unstable manifold is Hölder continuous, and unstable manifolds have uniformly bounded curvature [9, 10], hence once can easily see that

$$
\left|\Delta_{1}\right| \leq C \delta\left|\theta_{1}-\theta\right|^{\gamma}
$$


for some constants $C>0$ and $\gamma>0$ (in fact, $\gamma=1 / 3$ for our model; cf. [5, Corollary 5.30]). It remains to show that

$$
\lim _{\theta_{1} \rightarrow \theta} \limsup _{\delta \rightarrow 0}\left|\Delta_{2}\right| / \delta=0,
$$

i.e., the contribution from unstable manifolds crossing just one rectangle is negligible.

To estimate $\Delta_{2}$, denote by $F(y)=\mu(\cup W:|W|<y)$ be the measure of all the unstable manifolds of length $<y$. Then eq.(38) can be written as

$$
F(2 \delta)+\int_{2 \delta}^{\infty} \frac{2 \delta}{y} d F(y) \leq C \delta .
$$

Dividing by $\delta$ and taking the limit $\delta \rightarrow 0$ gives

$$
2 \int_{0}^{\infty} \frac{d F(y)}{y}<C
$$

(see also [5, Exercise 7.15]). Now let $L_{\theta}=\{(\theta, \psi): \psi \in[-\pi / 2, \pi / 2]\}$ denote the line in $\mathcal{M}$ with the fixed $\theta$ coordinate. Denote

$$
F_{\theta, \theta_{1}}(y)=\mu\left(\cup W:|W|<y, W \text { terminates between } L_{\theta} \text { and } L_{\theta_{1}}\right) .
$$

Then we have

$$
\limsup _{\delta \rightarrow 0} \frac{\left|\Delta_{2}\right|}{\delta} \leq \int_{0}^{\infty} \frac{d F_{\theta, \theta_{1}}(y)}{y}
$$

Now, as $\theta_{1}$ and $\theta$ get closer together, $F_{\theta, \theta_{1}}(y)$ monotonically decreases for each fixed $y>0$. Moreover, we have

$$
\lim _{\theta_{1} \rightarrow \theta} F_{\theta, \theta_{1}}(y)=0 \quad \forall y>0
$$

because the union of unstable manifolds terminating exactly on the line $L_{\theta}$ has $\mu$-measure zero. To see this observe that unstable manifolds terminate on singularity lines of the past iterations of the collision map $T$, i.e., on singularity lines of $T^{-n}, n>0$. These lines intersect the line $L_{\theta}$ at countably many points thus there are at most countably many unstable manifolds terminating on $L_{\theta}$. Finally each individual unstable manifold has $\mu$-measure zero, as is guaranteed by the Poincaré recurrence theorem. Now combining eq.(41)-eq.(43) proves eq. (40).

In smooth hyperbolic systems without singularities all unstable manifolds are long enough so that $\Delta_{2}=0$. Then the density $\rho$ is not only continuous, but Hölder continuous, according to eq.(39). We believe that in our MH model, too, the main contribution to the structure of $\rho$ comes from $\Delta_{1}$, so that $\rho$ is also Hölder continuous, but our estimate eq.(40) on $\Delta_{2}$ is too poor to prove that.

The above argument applies to projections of SRB measures onto some coordinates (transversal to stable and unstable directions). If, instead of projections, we integrate smooth functions like in eq.(14), then those functions can be incorporated into conditional densities of the SRB measure on unstable manifolds, and the argument will work for that situation, too.

\section{B Derivation of Green-Kubo formulas}

The derivation of eqs.(9,10) and (18) is based on a Kawasaki-type formula used in linear response theory, see [4]. For a small external field $E$ and the corresponding SRB measure $\mu_{E}$, we can integrate any bounded piecewise Hölder continuous function $f_{E}$ on $\mathcal{M}$ as follows:

$$
\mu_{E}\left(f_{E}\right)=\mu_{0}\left(f_{E}\right)+\sum_{n=1}^{\infty} \mu_{0}\left(\left(f_{E} \circ T_{E}^{n}\right)\left(1-e^{-E \Delta_{E}}\right)\right),
$$


where $\Delta_{E}$ denotes the displacement of the particle in the direction of the field (i.e., in the positive $x$ direction) during its free flight to the next collision. More precisely, for $(\theta, \psi) \in \mathcal{M}$ we set

$$
\Delta_{E}(\theta, \psi)=\int_{0}^{\tau_{E}(\theta, \psi)} \cos \left(\Phi_{t}^{E}(\theta, \psi)\right) d t
$$

where we use this definition to avoid the ambiguity on the difference between two points on a torus (periodic boundary conditions). The above Kawasaki-type formula eq.(44) is derived in [4, Eq. (16)].

The function $f_{E}$ may depend on the field $E$, but it must have a limit $f_{0}=\lim f_{E}$ as $E \rightarrow 0$. Since $T_{0}=\lim _{E \rightarrow 0} T_{E}$ and $\tau_{0}=\lim _{E \rightarrow 0} \tau_{E}$, as well as $\Delta_{0}=\lim _{E \rightarrow 0} \Delta_{E}=\tau \cos \phi$, a first order Taylor expansion of the infinite sum in eq.(44) gives

$$
\mu_{E}\left(f_{E}\right)=\mu_{0}\left(f_{E}\right)+E \sum_{n=1}^{\infty} \mu_{0}\left(\left(f_{0} \circ T_{0}^{n}\right) \Delta_{0}\right)+o(E),
$$

see [4, Eq. (17)]. The infinite sum in the above equation converges because $\mu_{0}\left(\left(f_{0} \circ T_{0}^{n}\right) \Delta_{0}\right) \rightarrow$ $\mu_{0}\left(f_{0}\right) \mu_{0}\left(\Delta_{0}\right)=0$ exponentially fast as $n \rightarrow \infty$ for $f_{0}$ Hölder continuous (decay of correlations) and $\mu_{0}\left(\Delta_{0}\right)=0$. Note that this sum is independent of $E$, hence the second term is linear in $E$. The first term $\mu_{0}\left(f_{E}\right)$ will be handled separately for each $f_{E}$.

\section{B.1 Derivation of Eqs.(9) and (10)}

We first derive eq.(9) for the local density $n_{E}(\mathbf{x})$, which can be represented, according to eq.(7), by

$$
\delta^{2} n_{E}(\mathbf{x})=\mu_{E}\left(J_{A}^{E}\right)+o\left(\delta^{2}\right)
$$

where $A=\left\{\mathbf{x}^{\prime}:\left\|\mathbf{x}^{\prime}-\mathbf{x}\right\|_{\infty} \leq \delta / 2\right\}$ denotes the square in $Q$ with side $\delta$ centered on $\mathbf{x}$. We fix a small $\delta>0$ and will estimate the integral in eq.(47).

It is convenient to extend the space $\mathcal{M}$ by adding the sides of the square $A$ to $\partial Q$. In other words, every time a trajectory crosses the boundary of $A$ and enters or exits $A$ we register a 'virtual collision' (the trajectory does not actually change its direction, so the collision has no effect on the trajectory of the particle, i.e. $\partial A$ plays the role of 'transparent walls'). Adding transparent walls with virtual collisions is a useful trick in the study of billiards.

By adding this transparent wall the phase space of the system is thus extended from $\mathcal{M}=$ $[0,4 \pi] \times[-\pi / 2, \pi / 2]$ to $\mathcal{M}_{A}=[0,4 \pi+4 \delta] \times[-\pi / 2, \pi / 2]$ where $\theta \in[4 \pi, 4 \pi+4 \delta]$ parametrize $\partial A$. Consistently we must replace the map $T_{E}$ with the new map $T_{E, A}$ constructed as in section 1, the SRB measure $\mu_{E}$ with $\mu_{E, A}$ defined as in eq.(3) and the function $J_{A}^{E}$ with $J_{A}^{E, A}$ defined as in eq.(6) . The notation $J_{A}^{E, A}$ helps keeping track of the fact the $A$ appears both in the indicator function appearing in eq.(6) and in the phase space $\mathcal{M}_{A}$ on which $J_{A}^{E, A}$ is defined. From this follows that $J_{A}^{E, A} \neq 0$ if and only if $\theta$ represents a collision taking place on $\partial A$ and the outgoing velocity points inside $A$. Indeed, $I_{A}\left(\mathbf{X}_{t}(\theta, \psi)\right) \neq 0$ if and only if $\mathbf{X}_{t}(\theta, \psi) \in A$. But in this case the last collision of the trajectory was with $\partial A$ and the velocity was pointing inside $A$. get

Clearly eq. (46) remains true if we replace $\mu_{E}$ with $\mu_{E, A}$. We apply it to $f_{E}=J_{A}^{E, A}$ and

$$
\delta^{2} n_{E}(\mathbf{x})=\mu_{0, A}\left(J_{A}^{0, A}\right)+\mu_{0, A}\left(\chi_{A}^{E}\right)+E \sum_{n=1}^{\infty} \mu_{0, A}\left(\left(J_{A}^{0, A} \circ T_{0, A}^{n}\right) \Delta_{0, A}\right)+o(E)
$$

where $\Delta_{0, A}$ is defined as in eq.(45) and $\chi_{A}^{E}=J_{A}^{E, A}-J_{A}^{0, A}$. By direct calculations we get

$$
\mu_{0, A}\left(J_{A}^{0, A}\right)=\frac{\delta^{2}}{\operatorname{Area}(Q)}=\delta^{2} n_{0}(\mathbf{x}) .
$$


Observe that, for every $E, J_{A}^{E, A}=O(\delta)$ while $\mu_{0, A}\left(\operatorname{supp}\left(J_{A}^{E, A}\right)\right)=O(\delta)$. Moreover, from eq.(11) we have that $\left|\mathbf{X}_{t}^{E}(\theta, \psi)-\mathbf{X}_{t}^{0}(\theta, \psi)\right|=O\left(E t^{2}\right)$ so that $J_{A}^{E, A}-J_{A}^{0, A}=O\left(\delta^{2} E\right)$. Thus even though the term $\mu_{0, A}\left(\chi_{A}^{E}\right)=O\left(E \delta^{3}\right)$ is linear in $E$, its contribution vanishes in the limit $\delta \rightarrow 0$ and we will ignore it. We finally arrive at

$$
n_{E}(\mathbf{x})=n_{0}(\mathbf{x})+\delta^{-2} E \sum_{n=1}^{\infty} \mu_{0, A}\left(\left(J_{A}^{0, A} \circ T_{0, A}^{n}\right) \Delta_{0}\right)+o(E) .
$$

Observe that a trajectory originating from a point $\mathbf{x}$ gives a non zero contribution to the $n$-th term in the sum only if its $n$-th collision is with $\partial A$ and the trajectory enters $A$. Since $E$ and $\delta$ are small, this implies that the $n-1$-th collision was with a non-virtual obstacle. We can thus rewrite eq.(49) as

$$
d(\mathbf{x})=\delta^{-2}\left(\mu_{0}\left(J_{A}^{0} \Delta_{0, A}\right)+\sum_{n=1}^{\infty} \mu_{0}\left(\left(J_{A}^{0} \circ T_{0}^{n}\right) \Delta_{0}\right)\right)
$$

where we have neglected the trajectories that collide more than once with $A$, since they contribute $O\left(\delta^{3}\right)$ to the integral, and the difference between $\bar{\tau}_{E}$ and $\bar{\tau}_{E, A}$, appearing in $J_{A}^{E, A}$, since it is $O(E)$ and thus does not contribute at first order.

We still have to discuss the limit $\delta \rightarrow 0$ in eq.(50). This limit is non trivial since, although the correlations appearing in the infinite sum decay exponentially for every $A$, we need to show that such a decay is uniform in $\delta$. To show this we can take the limit $\delta \rightarrow 0$ term by term in the sum. Taking this into account we obtain eq.(9).

We can now derive eq.(10) for the local average velocity $\mathbf{v}_{E}(\mathbf{x})$. Similarly to eq.(47) we have

$$
\delta^{2} \mathbf{v}_{E}(\mathbf{x})=\frac{1}{n_{E}(\mathbf{x})} \mu_{E}\left(\mathbf{H}_{A}^{E}\right)+o\left(\delta^{2}\right),
$$

where

$$
\mathbf{H}_{A}^{E}(\theta, \psi)=\frac{1}{\bar{\tau}_{E}} \int_{0}^{\tau_{E}(\theta, \psi)}\left(\cos \left(\Phi_{t}^{E}(\theta, \psi)\right), \sin \left(\Phi_{t}^{E}(\theta, \psi)\right)\right) I_{A}\left(X_{t}^{E}(\theta, \psi)\right) d t
$$

and $A$ again denotes the square in $Q$ with side $\delta$ centered on $\mathbf{x}$. In this case we will not need to introduce virtual collisions with $A$ like we did for eq.(9). Applying eq.(44) we obtain

$$
\delta^{2} n_{E}(\mathbf{x}) \mathbf{v}_{E}(\mathbf{x})=\mu_{0}\left(\mathbf{H}_{A}^{E}\right)+E \sum_{n=1}^{\infty} \mu_{0}\left(\left(\mathbf{H}_{A}^{0} \circ T_{0}^{n}\right) \Delta_{0}\right)+o(E)
$$

To eliminate the first term $\mu_{0}\left(\mathbf{H}_{A}^{E}\right)$ we apply an antisymmetrization. Due to the invariance of $\mu_{E}$ we have $\mu_{E}\left(\mathbf{H}_{A}^{E}\right)=\mu_{E}\left(\mathbf{H}_{A}^{E} \circ T_{E}^{-1}\right)$, hence, by applying eq. (44) to $\mathbf{H}_{A}^{E} \circ T_{E}^{-1}$, we get

$$
\delta^{2} n_{E}(\mathbf{x}) \mathbf{v}_{E}(\mathbf{x})=\mu_{0}\left(\mathbf{H}_{A}^{E} \circ T_{E}^{-1}\right)+E \sum_{n=0}^{\infty} \mu_{0}\left(\left(\mathbf{H}_{A}^{0} \circ T_{0}^{n}\right) \Delta_{0}\right)+o(E)
$$

Next, let $\mathcal{J}: \mathcal{M} \rightarrow \mathcal{M}$ denote an involution defined by $\mathcal{J}(\theta, \psi)=C(\theta,-\psi)$. Due to the time reversibility of the perturbed dynamics we have $\mathcal{J} \circ T_{E}=T_{E}^{-1} \circ \mathcal{J}$, and therefore $\mathbf{H}_{A}^{E}=-\mathbf{H}_{A}^{E} \circ \mathcal{J} \circ T_{E}$. Also note that $\mu_{0}$ is invariant under both $T_{0}$ and $\mathcal{J}$. Thus if we add eq.(53) and eq.(54), the first terms cancel out.

Moreover, the time reversibility of the billiard dynamics implies $\Delta_{0}=-\Delta_{0} \circ \mathcal{J} \circ T_{0}$ and $\mathbf{H}_{A}^{0} \circ T_{0}^{n}=-\mathbf{H}_{A}^{0} \circ T_{0}^{-n} \circ \mathcal{J} \circ T_{0}$ for all $n$. Therefore,

$$
\left(\mathbf{H}_{A}^{0} \circ T_{0}^{n}\right) \cdot \Delta_{0}=\left[\left(\mathbf{H}_{A}^{0} \circ T_{0}^{-n}\right) \Delta_{0}\right] \circ \mathcal{J} \circ T_{0} .
$$


Thus adding eq.(57) and eq.(58) together gives

$$
\delta^{2} n_{E}(\mathbf{x}) \mathbf{v}_{E}(\mathbf{x})=\frac{1}{2} E \sum_{n=-\infty}^{\infty} \mu_{0}\left(\left(\mathbf{H}_{A}^{0} \circ T_{0}^{n}\right) \Delta_{0}\right)+o(E)
$$

Taking into account eq.(49) we get

$$
\mathbf{k}_{E}(\mathbf{x})=\frac{1}{2 \delta^{2} n_{0}(\mathbf{x})} \sum_{n=-\infty}^{\infty} \mu_{0}\left(\left(\mathbf{H}_{A}^{0} \circ T_{0}^{n}\right) \Delta_{0}\right)
$$

where, due to the time reversibility of the dynamics, the term for $n$ and $-n$ in the sum are equal. To obtain eq.(10) we have used that $\lim _{\delta \rightarrow 0} \delta^{-2} \mu_{0}\left(\left(\mathbf{H}_{A}^{0} \circ T_{0}^{n}\right) \Delta_{0}\right)=c\left(\rho_{n}^{c}\left(\Delta_{0}\right), \rho_{n}^{s}\left(\Delta_{0}\right)\right)$ with $c=n_{0}(\mathbf{x})$.

\section{B.2 Derivation of Eq.(18)}

To derive eq.(18), we apply eq.(46) to $f_{E}=J_{A(\phi, \delta)}^{E}$, which was defined in eq.(6) and where $A(\phi, \delta)$ is the set $\phi-\delta / 2 \leq \theta+\psi \leq \phi+\delta / 2$, i.e. the set of velocity vectors that make an angle $\phi^{\prime} \in[\phi-\delta / 2, \phi+\delta / 2]$. Note that when $E=0$, the trajectory is a straight line, so $f_{0}=\tau_{0} I_{A(\phi, \delta)}$.

As in eq. (47) we can write $\delta h_{E}(\phi)=\mu_{E}\left(J_{A(\phi, \delta)}^{E}\right)+o(\delta)$. We denote $\Delta_{\phi, \delta}=\Delta_{0} \cdot I_{A(\phi, \delta)}$ and recall that $\Delta_{0}=\tau_{0} \cos \phi$, so that $J_{A(\phi, \delta)}^{0} \cos \phi=\Delta_{\phi, \delta} / \bar{\tau}_{0}$. Thus eq. (46) becomes

$$
\delta \cos \phi h_{E}(\phi)=\mu_{0}\left(J_{A(\phi, \delta)}^{E}\right) \cos \phi+\frac{E}{\bar{\tau}_{0}} \sum_{n=1}^{\infty} \mu_{0}\left(\left(\Delta_{\phi, \delta} \circ T_{0}^{n}\right) \Delta_{0}\right)
$$

and, again due to the invariance of $\mu_{E}$, we get

$$
\delta \cos \phi h_{E}(\phi)=\mu_{0}\left(J_{A(\phi, \delta)}^{E} \circ T_{E}^{-1}\right) \cos \phi+\frac{E}{\bar{\tau}_{0}} \sum_{n=0}^{\infty} \mu_{0}\left(\left(\Delta_{\phi, \delta} \circ T_{0}^{n}\right) \Delta_{0}\right)
$$

and (denoting, for brevity, $\phi^{-}=\phi+\pi$ )

$$
\delta \cos \phi^{-} h_{E}\left(\phi^{-}\right)=\mu_{0}\left(J_{A\left(\phi^{-}, \delta\right)}^{E}\right) \cos \phi^{-}+\frac{E}{\bar{\tau}_{0}} \sum_{n=1}^{\infty} \mu_{0}\left(\left(\Delta_{\phi^{-}, \delta} \circ T_{0}^{n}\right) \Delta_{0}\right),
$$

In all the above formulas we have suppressed the $o(E \delta)$ terms. Time reversibility implies $J_{A(\phi, \delta)}^{E} \circ T_{E}^{-1}=J_{A\left(\phi^{-}, \delta\right)}^{E} \circ \mathcal{J}$, where $\mathcal{J}$ denotes the time reversal involution. Thus, if we add eq.(57) and eq.(58), their first terms cancel out. Similarly, we get $\Delta_{0}=-\Delta_{0} \circ \mathcal{J} \circ T_{0}$ and $\Delta_{\phi^{-}, \delta} \circ T_{0}^{n}=-\Delta_{\phi, \delta} \circ T_{0}^{-n} \circ \mathcal{J} \circ T_{0}$ for all $n$. Therefore,

$$
\left(\Delta_{\phi^{-}, \delta} \circ T_{0}^{n}\right) \cdot \Delta_{0}=\left[\left(\Delta_{\phi, \delta} \circ T_{0}^{-n}\right) \Delta_{0}\right] \circ \mathcal{J} \circ T_{0} .
$$

Thus adding eq.(57) and eq.(58) gives

$$
h_{E}(\phi)-h_{E}(\phi+\pi)=\frac{E}{\delta \bar{\tau}_{0} \cos \phi} \sum_{n=-\infty}^{\infty} \mu_{0}\left(\left(\Delta_{\phi, \delta} \circ T_{0}^{n}\right) \Delta_{0}\right)+o(E) .
$$

This is an infinite sum of correlations which decay exponentially fast [5]. 
In the case of the billiard shown in Figure 1 we have that, due to the symmetry of the system, $h_{E}(\phi+\pi)=h_{-E}(\phi)$ so that we get

$$
\begin{aligned}
a(\phi) & \left.=\frac{1}{2 \delta \bar{\tau}_{0} \cos \phi} \sum_{n=-\infty}^{\infty} \mu_{0}\left(\left(\Delta_{\phi, \delta} \circ T_{0}^{n}\right) \Delta_{0}\right)\right) \\
& =\frac{1}{2 \delta \bar{\tau}_{0} \cos \phi} \sum_{n=-\infty}^{\infty} \mu_{0}\left(\Delta_{\phi, \delta} \cdot\left(\Delta_{0} \circ T_{0}^{n}\right)\right)
\end{aligned}
$$

where we used the invariance of $\mu_{0}$ under $T_{0}$. Finally using the relation $\Delta_{\phi, \delta}=\tau_{0} I_{A(\phi, \delta)} \cos \phi$ and taking the limit $\delta \rightarrow 0$ gives eq.(19).

\section{Isotropy of the Collision Time}

As a motivation for our definition of $\mu_{0}^{\phi}\left(\tau_{0}\right)$, we define a directional mean free time as follows. Given $\phi \in[0,2 \pi]$ and $\delta>0$, let $I=I_{A(\phi, \delta)}$ be as in Section B.2. Due to ergodicity, we have

$$
\lim _{n \rightarrow \infty} \frac{\sum_{i=0}^{n-1} \tau_{0}\left(T_{0}^{i}(\theta, \psi)\right) I\left(T_{0}^{i}(\theta, \psi)\right)}{\sum_{i=0}^{n-1} I\left(T_{0}^{i}(\theta, \psi)\right)}=\frac{\mu_{0}\left(\tau_{0} I\right)}{\mu_{0}(I)}
$$

for almost every $(\theta, \psi) \in \mathcal{M}$, and we call the limit (if it exists)

$$
\bar{\tau}_{\phi}=\lim _{\delta \rightarrow 0} \frac{\mu_{0}\left(\tau_{0} I\right)}{\mu_{0}(I)}
$$

the directional mean free time (corresponding to the angle $\phi$ ). Now arguing as in Section B.2 we get

$$
\lim _{\delta \rightarrow 0} \delta^{-1} \mu_{0}\left(\tau_{0} I\right)=\int \tau(\phi-\theta, \theta) \cos (\phi-\theta) \chi(\theta) d \theta
$$

and

$$
\lim _{\delta \rightarrow 0} \delta^{-1} \mu_{0}(I)=\int \cos (\phi-\theta) \chi(\theta) d \theta=Z_{\phi},
$$

recall eq.(17). Therefore

$$
\bar{\tau}_{\phi}=\mu_{0}^{\phi}\left(\tau_{0}\right)
$$

is the conditional expectation of $\tau$ defined in Section 2.2

Now it is easy to see that $\cos (\phi-\theta) \chi(\theta) d \theta$ is the length element in the direction orthogonal to the outgoing velocity vector (i.e., in the direction $\phi+\pi / 2$ ). Therefore the integral in eq.61) is equal to the area of the billiard table, which is $1-\pi\left(r_{1}^{2}+r_{2}^{2}\right)$ in our case. Thus

$$
\bar{\tau}_{\phi}=\mu_{0}^{\phi}\left(\tau_{0}\right)=\frac{1-\pi\left(r_{1}^{2}+r_{2}^{2}\right)}{2\left(r_{1}+r_{2}\right)},
$$

which is constant (independent of $\phi$ ).

The above argument generalizes to any Sinai billiard with convex obstacles $B_{1}, \ldots, B_{p}$. For each obstacle $B_{k}$ and angle $\phi$ we denote by width ${ }_{\phi}\left(B_{k}\right)$ the "width" of $B_{k}$ in the direction

orthogonal to $\phi$, i.e., the length of the projection of $B_{k}$ onto a line orthogonal to all velocities running at the angle $\phi$. Then by the above argument we have

$$
\bar{\tau}_{\phi}=\frac{\operatorname{Area}(Q)}{\sum_{k} \operatorname{width}_{\phi}\left(B_{k}\right)} .
$$


This formula holds for each $\phi$ except directions in which billiard trajectories can run indefinitely without collisions. If the horizon is finite, no such trajectory exists, and eq.(63) holds for every $\phi$. If the obstacles are circular disks, as they are in our studies, the "width" of $B_{k}$ is just its diameter, and comparing eq.(63) with eq.(64) we see that $\bar{\tau}_{\phi}$ is constant, i.e., independent of $\phi$.

It is not hard to see that averaging $\bar{\tau}_{\phi}$ over $\phi$ gives

$$
\frac{1}{2 \pi} \int_{-\pi}^{\pi} \bar{\tau}_{\phi} d \phi=\bar{\tau}_{0}=\mu_{0}\left(\tau_{0}\right)
$$

the classical (unconditional) mean free path, which is known to be

$$
\bar{\tau}_{0}=\frac{\pi \cdot \operatorname{Area}(Q)}{\operatorname{length}(\partial Q)}
$$

see [5, Section 2.13]. If $\bar{\tau}_{\phi}$ is constant, then of course $\bar{\tau}_{\phi}=\bar{\tau}_{0}$ for all $\phi$.

\section{References}

[1] F. Bonetto, D. Daem, J. Lebowitz, "Properties of Stationary Nonequilibrium States in the Thermostatted Periodic Lorentz Gas I: The One Particle System", JSP 35-60, 101 2000

[2] F. Bonetto, D. Daem, J. Lebowitz, V. Ricci, "Properties of stationary nonequilibrium states in the thermostatted periodic Lorentz gas: The multiparticle system", PRE 05124, 652002

[3] F. Bonetto, J. Lebowitz, "Nonequilibrium Stationary Solution of Thermostatted Boltzmann Equation in a Field", New Trends in Statistical Physics: Festschrift in honor of Leopoldo Garcia-Colin's 80th birthday 27-36, (2009) .

[4] N. Chernov, G. Eyink, J. E. Lebowitz, and Ya. G. Sinai, "Steady state electric conductivity in the periodic Lorentz gas", Commun. in Math. Phys. 569-601, 154 (1993).

[5] N. Chernov and R. Markarian, "Chaotic Billiards", Mathematical Surveys and Monographs, 127, AMS, Providence, RI, 2006. (316 pp.)

[6] T. Yamada and K. Kawasaki, "Nonlinear effects in the shear viscosity of a critical mixture", Prog. Theor. Phys. 1031-1051, 38 (1967).

[7] H. Spohn, Large scale dynamics of interacting particles Springer-Verlag, New York, (1991).

[8] D. Evans, G.P. Morris, Statistical Mechanics of Nonequilibrium Fluids Academic Press, San Diego, (1990)

[9] N. Chernov, "Sinai billiards under small external forces", Ann. Henri Poincare, 197-236 2 (2001).

[10] H.K. Zhang, "Current in periodic Lorentz gases with twists" Comm. Math. Phys., to appear.

[11] P. Frederickson, J.L. Kaplan, E.D. Yorke, J.A. Yorke, "The Lyapunov Dimension of Strange Attractors", J. Diff. Eq. 185-207, 49 (1983).

[12] B. Moran and W. G. Hoover, "Diffusion in the periodic Lorentz billiard", J. Stat. Phys., 709-726, 48 (1987). 
[13] J. P. Eckmann and D. Ruelle, "Ergodic theory of chaos and strange attractors", Rev. Mod. Phys. 617-656, 57 (1985).

[14] N. Chernov and D. Dolgopyat, "Brownian Brownian Motion-1", Memoirs AMS 198, 2009, (193 pp)

[15] Y.H. Du, Order Structure and Topological Methods in Nonlinear Partial Differential Equations, World Scientific (2006)

[16] K. Deimling, Nonlinear Functional Analysis, Springer-Verlag (1985). 